\title{
La vallée de la Seiche et la haute vallée de Vilaine du début du Néolithique au Chalcolithique : bilan et perspectives
}

The Valley of Seiche and the high Valley of Vilaine since Neolithic to

Chalcolithic: summary and prospects

Das Tal der Seiche und das obere Tal der Vilaine vom Beginn des Neolithikums

bis zum Chalkolithikum: Bilanz und Forschungsperspektiven

El valle del Seiche y la cuenca alta del Vilaine desde los inicios del Neolítico al Calcolítico: balance y perspectivas

\section{Gwenolé Kerdivel}

\section{OpenEdition}

Journals

Édition électronique

URL : https://journals.openedition.org/rao/6775

DOI : $10.4000 /$ rao. 6775

ISSN : 1775-3732

Éditeur

Presses universitaires de Rennes

Édition imprimée

Date de publication : 21 décembre 2021

Pagination : 99-123

ISBN : 978-2-7535-8668-0

ISSN : 0767-709X

\section{Référence électronique}

Gwenolé Kerdivel, «La vallée de la Seiche et la haute vallée de Vilaine du début du Néolithique au

Chalcolithique : bilan et perspectives », Revue archéologique de l'Ouest [En ligne], 37 | 2021, mis en ligne le 21 décembre 2021, consulté le 11 octobre 2022. URL : http://journals.openedition.org/rao/6775 ;

DOI : https://doi.org/10.4000/rao.6775 


\title{
La vallée de la Seiche et la haute vallée de Vilaine du début du Néolithique au Chalcolithique : bilan et perspectives
}

\author{
The Valley of Seiche and the high Valley of Vilaine since Neolithic to Chalcolithic: summary \\ and prospects
}

\author{
Gwenolé Kerdivel \\ Docteur en archéologie et archéosciences, Groupe de Recherches Archéologiques de la Mayenne, co-gérant du bar-tabac Le Temps de l'Harmonie 42 rue du Lac, \\ 35210 Châtillon-en-Vendelais (gwenole@ramirezkerdivel.eu)
}

\begin{abstract}
Résumé: Cet article fait le point sur le Néolithique de la haute vallée de Vilaine et de la vallée de la Seiche, en incluant celle du Blosne, toutes trois situées à l'est de la Bretagne, au cœur du Massif armoricain. À cette occasion, plusieurs découvertes sont signalées : quelques indices $\mathrm{du}$ Villeneuve-Saint-Germain, une possible sépulture type Passy, un lieu d'extraction de microgranite et de façonnage de hache dans ce matériau daté du Néolithique final, de nombreuses lames de hache largement inédites, etc. La mise en contexte environnementale dans une dynamique chronologique permet de montrer que les données disponibles s'inscrivent largement dans ce que l'on connaît aux marges du Massif armoricain avec une augmentation du nombre de gisements dans la dernière phase du Néolithique et une pression toujours croissante sur l'environnement. Le tout permet surtout de voir en creux l'étendue de ce que l'on ne sait pas et de dessiner des projets pour l'avenir.

Abstract: This paper reviews the Neolithic of the upper Vilaine valley and the Seiche valley, including the Blosne valley, all three located in eastern Brittany, in the heart of the Armorican Massif. Discoveries include some evidence of Villeneuve-Saint-Germain sites, a possible Passy-type burial site, a microgranite extraction site and an axe fashioned from this material, dating to the Final Neolithic, and numerous mostly unpublished axe blades. The environmental context in a chronological dynamic shows that the available data are largely in line with what is known at the margins of the Armorican Massif, with an increase in the number of deposits in the last phase of the Neolithic and ever-increasing pressure on the environment. Above all, it allows us to see the extent of what we do not know and to draw up plans for the future.
\end{abstract}

Mots clés: Bretagne, Néolithique, hache polie, habitat, menhir, prospection, archéologie spatiale, modèle de peuplement, occupation de l'espace.

Keywords: Brittany, Neolithic, stone axes, settlement, menhir, prospection, model of populating, spatial archaeology, occupation of the space.

Longtemps plébiscités sous la forme de mémoires universitaires, les inventaires et analyses des données archéologiques locales se font plus rares... Or, les approches à grande échelle méritent d'être explorées; c'est d'ailleurs l'une des pistes que nous ouvrions il y a déjà quelques années en conclusion d'un travail à petite échelle sur le Néolithique des marges du Massif armoricain (Kerdivel, 2012). Si les synthèses sur le Néolithique en Bretagne sont rares, c'est encore plus le cas des approches spatiales desdites données. Et si c'est plus par hasard que par nécessité - nous y résidons - que le choix du secteur étudié s'est porté sur la haute vallée de la Vilaine (909 km² environ) et la vallée de la Seiche ( $824 \mathrm{~km}^{2}$ environ), la situation de ce secteur au centre du Massif armoricain en amont d'une de ses vallées les plus importantes ouvre d'intéres- santes perspectives comparatives avec le reste de notre travail. Plus précisément, notre analyse porte sur ces deux vallées mais aussi sur la vallée du Blosne, afin d'en faire un secteur cohérent, ce qui porte l'ensemble du territoire exploré à $1809 \mathrm{~km}^{2}$ (fig. 1).

\section{LE CADRE GÉOLOGIQUE ET GÉOGRAPHIQUE}

Géologiquement (fig. 2), ces deux vallées recoupent essentiellement les formations briovériennes du domaine centre-armoricain, qui se caractérisent par une faible structuration et ne présentent guère de formations majeures : s'y retrouvent des grauwackes, des schistes tachetés et autres cornéennes (Trautmann et al., 1994). 


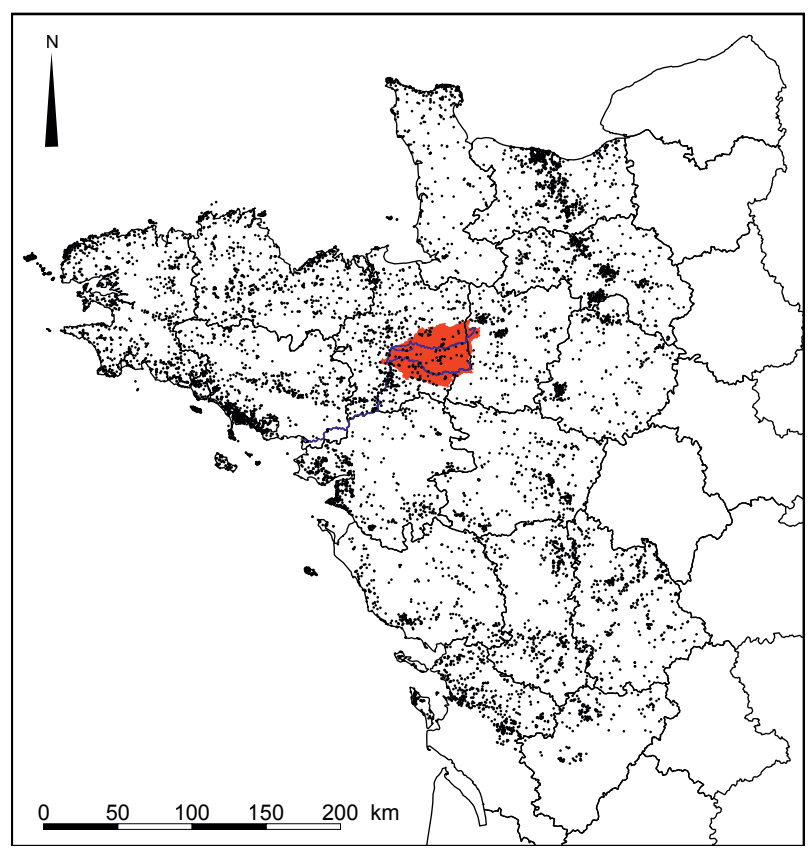

Figure 1 : Carte de situation générale du secteur étudié au sein de l'Ouest de la France néolithique.

Figure 1: Map of the study area in western France Neolithic.

Seul dénote uniquement le massif granitique varisque du Pertre (Trautmann et Carn, 1997). Au sud, la formation ordovicienne de Pont-Réan se caractérise par des grès, des conglomérats et surtout des siltstones micacés pourpres, les schistes rouges de la littérature archéologique
(Trautmann et al., 1994). Au nord et ponctuellement au sud, la formation ordovicienne de grès armoricain se caractérise par des conglomérats et des grès-quartzites blancs (Trautmann et al., 2011); au nord, les formations ordoviciennes de Saint-Germain-sur-Ille se caractérisent par des grès micacés verdâtres à intercalations de siltites noires, celle de La Lande-Murée par des grès-quartzites blancs ou gris-sombre pyriteux, celle d'Andouillé par des siltites noires et celle de Gahard par des grès quartzitiques noirs à gris (Trautmann et al., 2011). Au nord, le briovérien du domaine nord-armoricain peut localement être métamorphisé, notamment au contact des granodiorites à biotite et à cordiérite. À ces principaux terrains, il faut ajouter ponctuellement des roches filoniennes de type dolérite, microgranite et quartz, ainsi que des formations pliocènes et éocènes, essentiellement composées de sables et d'argiles au sud de Rennes (35).

Ce socle géologique influence un paysage aux formes plutôt monotones. Ainsi, les vallées de la Seiche et de la Vilaine recoupent une vaste pénéplaine, composée de plateaux divers, recoupés par de multiples talwegs peu encaissés. Elle est encadrée au sud par un relief appalachien (formation de Pont-Péan) qui court de la forêt de La Guerche-de-Bretagne jusqu’à Guichen (35) et au nord par les reliefs moutonnés des collines du Pays de Vitré (35).

Les sols ont sur l'ensemble du territoire une texture monotone, où limons et sables abondent, donnant des sols plutôt séchants ou battants, mais souvent profonds.

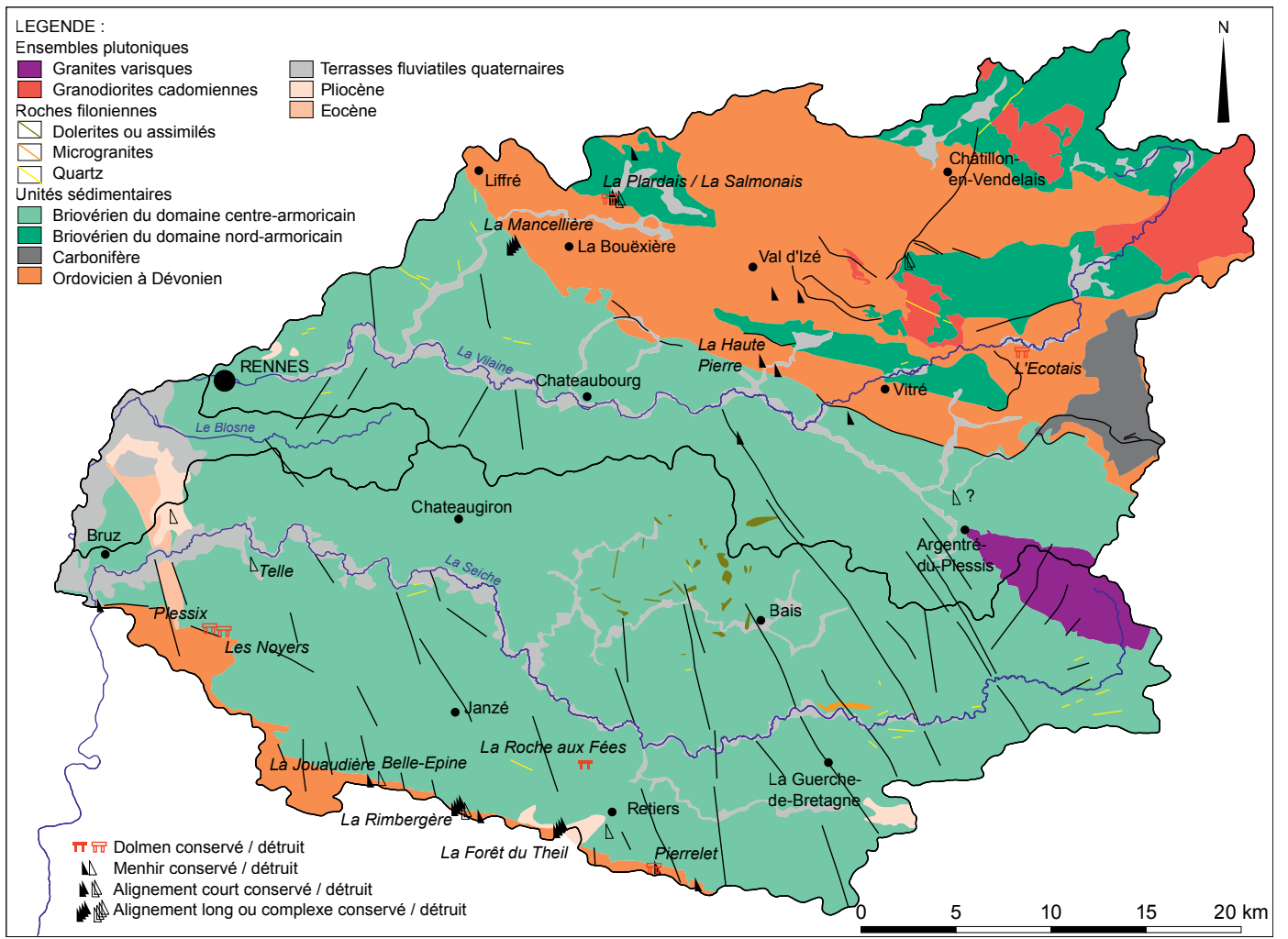

Figure 2 : Carte géologique simplifiée du secteur étudié et distribution des monuments mégalithiques connus.

Figure 2: Geological map (simplified) of the study area indicating the known megalithic monuments. 
L'acidité et l'hydromorphie divisent en deux le secteur étudié avec des concentrations plutôt à l'est qu'à l'ouest.

Il est difficile d'imaginer le paysage durant les presque 3000 ans que dure le Néolithique et le Chalcolithique. Les quelques données disponibles évoquent un paysage plutôt fermé (Gaudin, 2004). Il faut regretter ici l'absence d'analyses paléoenvironnementales recoupant la période Atlantique dans ces deux vallées alors qu'il existe un potentiel comme le montrent les sondages faits à Villaumur à Pocé-les-Bois (35) lors des travaux préalables au barrage de la Cantache et dans lesquels des végétaux anciens étaient conservés (Beuchet, 1993). Si aujourd'hui, la Vilaine n'est navigable que jusqu'à Rennes grâce aux nombreux aménagements d'écluses, elle devait l'être très largement en pirogue presque jusqu'à sa source à Juvigné (53). Sur toute sa longueur, la Seiche est elle aussi navigable sur de petites embarcations presque jusqu'au Pertre (35). Les gués et autres passages transversaux sont évidemment inconnus pour la préhistoire.

\section{LE CORPUS DE GISEMENTS ÉTUDIÉS}

Le secteur examiné regroupe 121 gisements néolithiques, dont $60 \%$ sont localisés de manière fiable $(\mathrm{n}=75)$. Afin de limiter certaines redondances et de laisser à leurs inventeurs la possibilité de publier leurs travaux issus de leurs fouilles archéologiques, nous limiterons essentiellement nos illustrations aux seuls éléments nouveaux que nous avons collectés.

Nous avons opté pour la nomenclature développée dans notre thèse (Kerdivel, 2012, p. 39-42). Ainsi, nous parlons de manière neutre de "gisement " comme un équivalent à " entité archéologique " qu'utilise la Carte archéologique. Les gisements se divisent en "sites » et en " indices de site ». Les premiers se divisent en " funéraire " et " non funéraire "; les seconds en "indice de site avec structure " (quand celle-ci se trouve en bord de décapage par exemple), en "mobilier épars » ou en "objet isolé ». Ainsi, les sites funéraires sont au nombre de huit : du monument mégalithique à la sépulture simple. Les sites non funéraires et non mégalithiques sont au nombre de 10. Les architectures de pierres dressées complexes sont au nombre de 7 et les menhirs seuls au nombre de 17. Les indices de site avec structures en creux sont au nombre de 9. Les indices de site avec seulement du mobilier en surface sont au nombre de 22 et les mobiliers isolés de 48 .

\section{Les gisements datés}

\section{Le début du Néolithique (3 gisements)}

Le Villeneuve-Saint-Germain, présent ailleurs en Illeet-Vilaine, n'est connu que ponctuellement sous la forme de mobilier épars, tous dans la vallée de la Seiche. À l'îlot Floratrait à Noyal-Châtillon-sur-Seiche (35), B. Simier (Inrap) a découvert au cours du décapage d'une villa antique une anse à ensellement médian décoré d'un décor arciforme et d'un bouton qui renvoie à ce groupe culturel (Simier, 2016). C'est aussi le cas des 128 tessons découverts par L. Aubry (Inrap) dans une des tranchées de la zone économique du bois de Teillay à Janzé (35). Ces tessons correspondent à au moins trois vases, découverts dans un niveau de colluvions portant des décors similaires à celui de l'îlot Floratrait (Aubry, 2017). Le fait qu'il s'agisse de niveaux de colluvions laisse à penser au fouilleur qu'un site se trouve en amont. Par contre, plus loin, dans la vallée de la Seiche, sur le site de Bellevue à Piré-sur-Seiche (35), G. Leroux (Inrap) a découvert un ensemble de structures très arasées (Leroux, 2015). Aucun plan particulier n'a pu être mis en évidence mais deux alignements de trois poteaux suggèrent un plan trapézoïdal typique de cette époque, mais bien difficile à attester. Le mobilier est rare mais désormais classique de cette culture avec des lames entières ou fragmentées et des burins en silex turonien, en silex du Cinglais et en silex tertiaire, matières importées.

\section{Le Néolithique moyen 1 (2 gisements)}

Au Heaulme à Visseiche (35), G. Leroux a repéré par avion un enclos allongé comparable à ceux du type Passy connus en Basse-Normandie, ce qui nous renverrait au début du Néolithique moyen (Leroux, 1997). Cette découverte est à prendre avec circonspection puisque ce type de structure est largement inconnu sur le Massif armoricain, hormis le cas de Saint-Loup-Lamairé (79; Germond, 2001), lui-même non fouillé. Toutefois, si le Cerny et le début du Néolithique moyen 1 sont rares, la découverte à Domloup (35), lieu-dit le Tertre, de deux fosses, dont les limites n'ont pas été perçues (faits 148.2 et 148.3), a livré à L. Aubry des tessons attribuables à cette culture, ainsi que des éclats lithiques et des pierres brûlées (Aubry, 2010). Au cours de la fouille d'une villa romaine importante au Bourg-Saint-Pair à Bais (35) par D. Pouille, a été observée une occupation sporadique qui se matérialise par $\mathrm{du}$ mobilier lithique - dont des lames en silex bathonien de Caen, des grattoirs sur lames, une limace, deux haches en fibrolite - attribué au Néolithique ancien ou au début du Néolithique moyen par S. Blanchet (Pouille, 2011).

\section{Le Néolithique moyen 2 (7 gisements)}

Le dolmen angevin de la Roche aux Fées à Essé (35) ne nécessite guère plus de présentation tant il est bien connu (Le Roux, 1998; Sellier, 2017). Les dolmens angevins restent mal datés du fait que peu d'entre eux ont été 
fouillés récemment d'une part et que leur chambre funéraire est souvent vide. Les fouilles du dolmen des Erves à Sainte-Suzanne (53) ou de Puyraveau à Saint-Léger-deMontbrun (79) ont cependant démontré la contemporanéité relative des dolmens angevins avec les dolmens à couloir du Néolithique moyen dont ils ne seraient qu'une variante (Letterlé, 1986; Joussaume et Laporte, 2006).

À Rannée (35), au lieu-dit la Sallerie, S. Barbeau (Inrap) a découvert au cours d'un diagnostic le fait 292 qui se compose de deux creusements : un de $2 \mathrm{~m}$ de largeur sur $0,90 \mathrm{~m}$ de profondeur et le second de $0,60 \mathrm{~m}$ par $0,60 \mathrm{~m}$. Son remplissage est complexe. Le mobilier se compose d'un vase à profil segmenté et d'un peson (Barbeau, 2014, p. 98-99). Aux Rimbaudières 3, sur la même commune et au cours de la même opération, une fosse F52 a livré une anse à ensellement médian (Barbeau, 2014, p. 76). La fouille de S. Sicard (Inrap) y a permis de découvrir, très dispersés sur la surface fouillée, deux foyers à pierres chauffées (F3 et F168) à 50 m l'un de l'autre, accompagnés de deux foyers en cuvette. Les six rejets de combustion sont dispersés plus loin. L'ensemble de ces structures est déconnecté de la découverte de S. Barbeau (Sicard, 2016) Leur datation est proposée sur la base de l'anse découverte dans la fosse F52. Des fragments de vases à profil segmenté ont aussi été découverts dans des fosses gauloises découvertes au cours des travaux préalables à la LGV au lieu-dit Mauzé à Torcé (35) et à la Fosse Levrette à Étrelles (35; Juhel, 2010).

Au Néolithique moyen lato sensu, on peut attribuer prudemment le site du Bertry à La Bouëxière (35) grâce à son couteau à dos et son armature tranchante (Onnée et al., 2000).

\section{Le Néolithique récent, final et Chalcolithique (acronyme NRF, 26 gisements)}

Bien qu'il faille distinguer plusieurs étapes pour la fin du Néolithique, l'état des connaissances régionales et des données locales induit de regrouper l'essentiel des données, puisqu'il est encore difficile de discriminer ces étapes par le matériel.

En ce qui concerne les sites funéraires, à la Plardais-la Salmonais à La Bouëxière (35, détruits), il existait une probable allée couverte d'une quinzaine de mètres de longueur (Bézier, 1883; Gaudin et al., 2000). Ce genre de monuments, s'il est construit vers le milieu du IV millénaire, va être utilisé sur la longue durée : les réoccupations campaniformes sont connues pour ce type de monument comme à Brécé (53; Bouillon, 1989).

Les cultures de la fin du Néolithique étant encore mal définies, plusieurs sites sont encore difficiles à situer. Le site de la Pinelière 2 à La Guerche-de-Bretagne (35) a été fouillé par S. Toron (Éveha) en 2015. Les vestiges très arasés du Néolithique sont un groupe de six fosses, dont seulement une a livré de la terre brûlée et des éléments d'un bol hémisphérique à fond plat, et un groupe de six trous de calage de poteau et d'une fosse (Toron, 2016). Les trous matérialisent le plan d'un petit bâtiment de $2,5 \mathrm{~m}$ de côté. Une datation radiocarbone sur charbon de bois (PRE-11801.16, 4241 $\pm 29 \mathrm{BP})$ permet de dater l'un des trous de poteau du premier quart du $\mathrm{III}^{\mathrm{e}}$ millénaire. Une datation semblable a été obtenue sur un charbon de bois prélevé dans le calage d'un bâtiment à deux pans d'environ $22 \mathrm{~m}$ de longueur et composé de neuf trous de calage de poteau, découvert à la Claraiserie à Ossé (35) par J. Le Gall (Inrap; Le Gall, 2015). À Cesson-Sévigné (35), au Petit Pré, S. Barbeau a découvert dans une fosse - un probable chablis - les restes d'au moins un vase du Néolithique final (Barbeau, 2011). À Bruz (35), à l'emplacement de la carrière de Cicé, deux locus différents ont été observés au gré des phases d'un vaste diagnostic. G. Leroux a découvert plusieurs structures (fossé et possibles trous de calage de poteau), associé à un épandage de mobilier de la fin du Néolithique (Leroux, 2003). De même, L. Aubry, $650 \mathrm{~m}$ plus loin, a découvert une fosse circulaire de $1,25 \mathrm{~m}$ de diamètre pour $0,35 \mathrm{~m}$ de profondeur ayant livré les restes d'un vase à profil rentrant et anse horizontale tri-perforée (Aubry, 2005). Plus prudemment, à Rennes (35), au lieu-dit la Poterie, D. Pouille (Inrap) rapporte l'existence de quelques fonds de structures archéologiques ayant livré un fragment de fusaïole et un éclat de silex retouché qu'il propose de dater de la fin du Néolithique (Pouille et Le Cloirec, 1992). Un vase à bouton de préhension, non dessiné, a été découvert par D. Pouille sur la ZAC des Grands Sillons à CorpsNuds (35) sans structure associée (Pouille, 2004). Le site antique de la Guyaumerais à Noyal-Châtillon-surSeiche (35) a été fouillé en sauvetage par A. Provost. Dans la couche 339, il signale un mobilier résiduel en plus du mobilier antique (Provost et al., 1985). Ce mobilier résiduel consiste en un mobilier lithique, dont des pointes de flèches pédonculées : nous ne l'avons pas examiné.

Le Chalcolithique et la culture campaniforme sont aussi représentés. Les Rives du Blosne à Chantepie (35) est un site découvert par G. Leroux et fouillé par S. Blanchet (Inrap) en 2003 (Blanchet, 2004). Il consiste en trois zones concentrant mobilier et structures. Le locus 3 est situé en bordure d'un affleurement de microgranite. Le locus 1 se matérialise par un niveau (US3) argilo-limoneux brun foncé et concentrant du mobilier néolithique en place mais bioturbé. À ce niveau sont associés cinq trous de calage de poteau et trois fosses. Le mobilier céramique présente des fonds plats, un fragment de cuillère et un bord de vase campaniforme; le lithique se compose de six éclats et d'une herminette en microgranite. Le locus 2 
se matérialise par le même niveau, deux trous de calage de poteau et quatre fosses. Le mobilier céramique abondant renvoie encore au Néolithique final, comme les deux fragments du même poignard en silex pressignien. Des blocs de microgranite en place dans ce niveau présentent des traces qui permettent de supposer l'existence d'une exploitation locale de ce matériau pour la fabrication de lames de hache. Enfin, une hache-marteau cordiforme a été découverte à $80 \mathrm{~m}$ à l'est (fig. 3). Sur le même site du Bourg Saint-Pair à Bais (35), D. Pouille (Inrap) a mis au jour un bâtiment en forme d'amande de $20 \mathrm{~m}$ de longueur pour 7,5 $\mathrm{m}$ de largeur à l'est et $2,5 \mathrm{~m}$ de largeur à l'ouest. Il se présente sous la forme d'une tranchée continue avec des surcreusements. Quelques autres structures lui sont associées. Les dates ${ }^{14} \mathrm{C}$ le placent entre 2460 et 2060 BCE (Pouille, 2011). D'autres constructions de ce type ont été découvertes en Bretagne et sont comparées aux dolmens en $V$ sur la base de leur plan (Blanchet $e t$ al., 2012). De même, à Torcé (35) - la Petite Gatellerie, deux tranchées de diagnostic ont permis à $\mathrm{F}$. Le Boulanger de découvrir une vingtaine de trous de calage de poteau très érodés et neuf fosses, dont certaines ont livré des tessons $\mathrm{du}$ Néolithique final très comparables aux découvertes des Rives du Blosne (Le Boulanger, 2012). À Visseiche (35) - la Grande Censerie, F. Le Boulanger a découvert au cours d'un diagnostic archéologique en 2002 plusieurs faits dans deux tranchées. Dans la tranchée 5 , trois dépressions, supposées naturelles, ont été fouillées et l'une (F14) a livré trois individus dont un gobelet campaniforme (Le Boulanger, 2002). Si la fonction des structures de Visseiche interroge, ce n'est pas le cas de celle des Rimbaudières 3, à Rannée (35), où $S$. Sicard a découvert une fosse (F156) de $1,10 \mathrm{~m}$ de longueur, pour $0,76 \mathrm{~m}$ de largeur et $0,12 \mathrm{~m}$ de profondeur : probable sépulture. Cette fosse était tapissée de blocs de grès et de schistes gréseux, comblement sur lequel reposaient les restes d'un gobelet campaniforme (Sicard, 2016). Au « set campaniforme " de la littérature (Guilaine et al., 2004) manque dans les deux cas le brassard d'archer; un exemplaire en schiste a justement été découvert au Breil Monfanil à Argentré-du-Plessis (35) avec une lame de hache en dolérite et une en silex (Meuret, 1986; 1993).
Figure 3 : Hache-marteau en roches vertes communes (dolérite?).

Figure 3: Axe-hammer in "common green stone" (dolerite?).

1 : La Boussardière à Cuillé (53), 2 : Les Rives du Blosne Locus 2 à Chantepie (35), 3 : Panlièvre à Amanlis (35), 4 : Le Plessis-Hubert à Chancé (35). Cette dernière est conservée au Laboratoire d'Anthropologie à Rennes.

1: La Boussardière à Cuillé (53), 2: Les Rives du Blosne Locus 2 à Chantepie (35), 3: Panlièvre à Amanlis (35), 4: Le Plessis-Hubert à Chancé (35). The latter is kept in Rennes's Anthropological Laboratory.

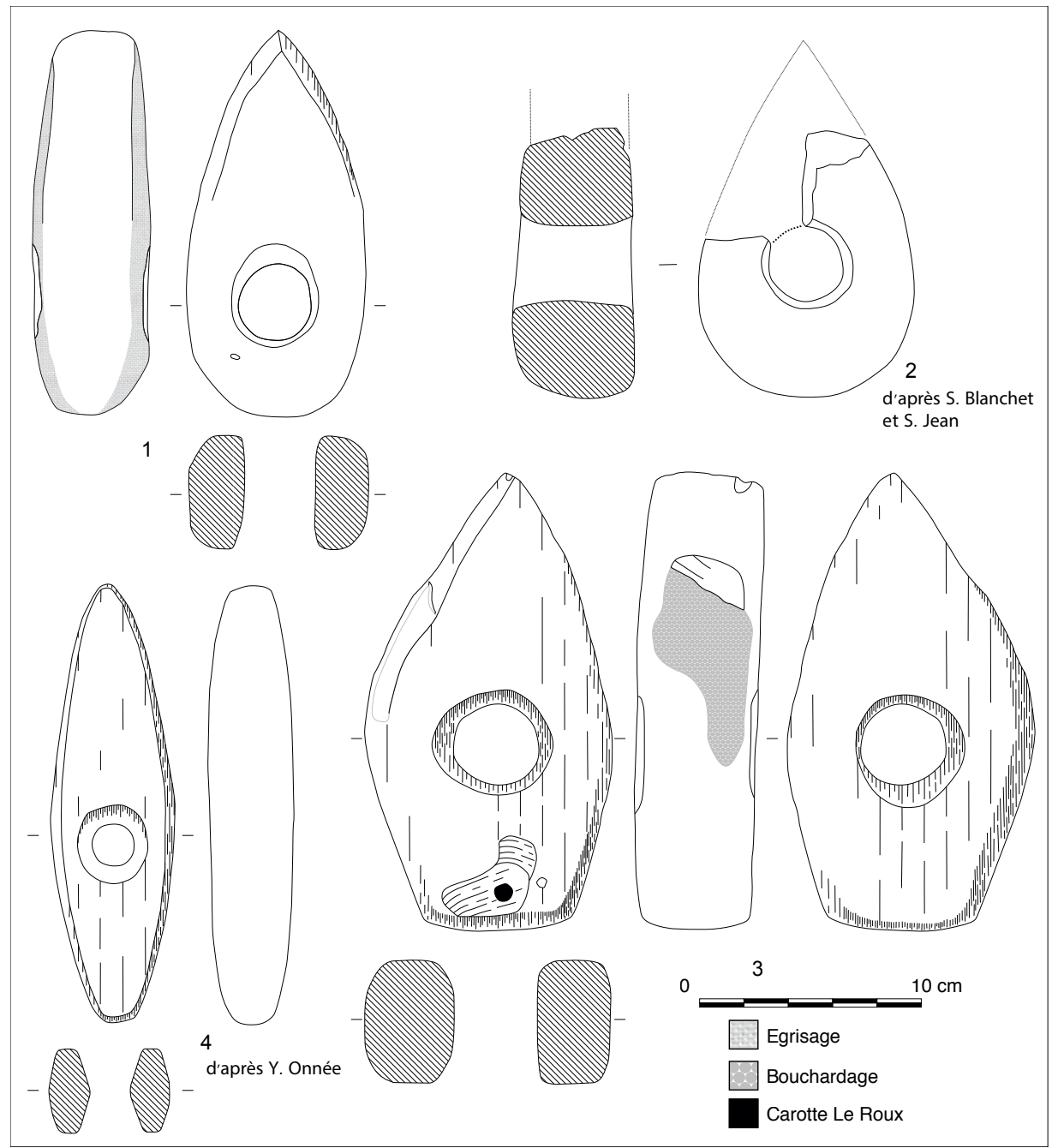


En plus de celui fragmenté découvert aux Rives du Blosne/Locus 2 (Blanchet, 2004, p. 26), un poignard entier en silex du Grand-Pressigny de $215 \mathrm{~mm}$ de longueur a été découvert à la Piaillerie à Janzé (35; Giot, 1960, p. 157). Un autre, court, a été découvert à la Fortinais à Louvigné-de-Bais (35; Corre, 2010). Autrement, un fragment a été découvert à la carrière des Vallons à Bais (35; Le Cloirec, 2005), deux fragments au Haut Champ Fleury à Liffré (35), un au Drugeon, un à la Touche Meslet et un à la Vieille Tarouanne à La Bouëxière (35; Onnée et al., 2000). Les quatre derniers signalent probablement des occupations plus grandes. La hache-marteau bipenne de Panlièvre à Amanlis (35), du groupe B type 3 défini par S. Barbier, peut être datée de cette époque (Priol, 1997 ; Barbier, 1992). D'autres haches-marteaux du groupe $A$ type 1 , en plus de celle du Blosne à Chantepie, sont connues au Plessis-Hubert à Chancé (35) et la Boussardière à Cuillé (35; Meuret, 1993; Giot, 1965; Kerdivel, 2019c; Barbier, 1992; Blanchet, 2004; fig. 3). Ce type n'est pas daté et très fréquent dans l'Ouest de la France (Barbier, 1992). Celle d'Ossé à Availles-sur-Seiche (35) n'a pas été retrouvée, de même que celle de Châtillonen-Vendelais (35), ce qui ne nous permet pas de savoir à quel type elles correspondent (De la Grancière et Harmois, 1916).

\section{Les gisements non datés}

\section{Les enceintes}

Néolithique ou âge du Bronze, il faut ajouter quatre enceintes curvilignes à fossés interrompus souvent doublés toutes découvertes par G. Leroux et de superficies variables (fig. 4) : le Mesnil à Champeaux (35; 1 ha; Beuchet, 1993), la Charonnière 2 à Saint-Aubin-desLandes (35; 3 ha; Hénaff, 2002), la Trappe à Boistrudan (35; 1 ha; Hénaff, 2002) et le Choiseul à Amanlis (35; 6 ha; Leroux, 1999). De toutes, celle de Boistrudan est la seule à avoir livré du mobilier archéologique attribuable au Néolithique lato sensu.

\section{Les dolmens à architecture indéterminée}

De même, on connaît quelques dolmens à architecture indéterminée (fig. 2). À l'Écotais à La ChapelleErbrée (35), le dolmen, dit Tombeau du Prêtre, est en quartzite, probablement détruit. Celui des Noyers à Orgères (35), dite Pierre-du-Diable, en schiste quartzeux, était composé de trois blocs et est détruit. Le dolmen de Pierrelet à Retiers (35) se situait à $13 \mathrm{~m}$ d'un menhir et était en schiste rouge (Bézier, 1883; Briard et al., 2004). Un autre type de monument au Plessix à Orgères (35)

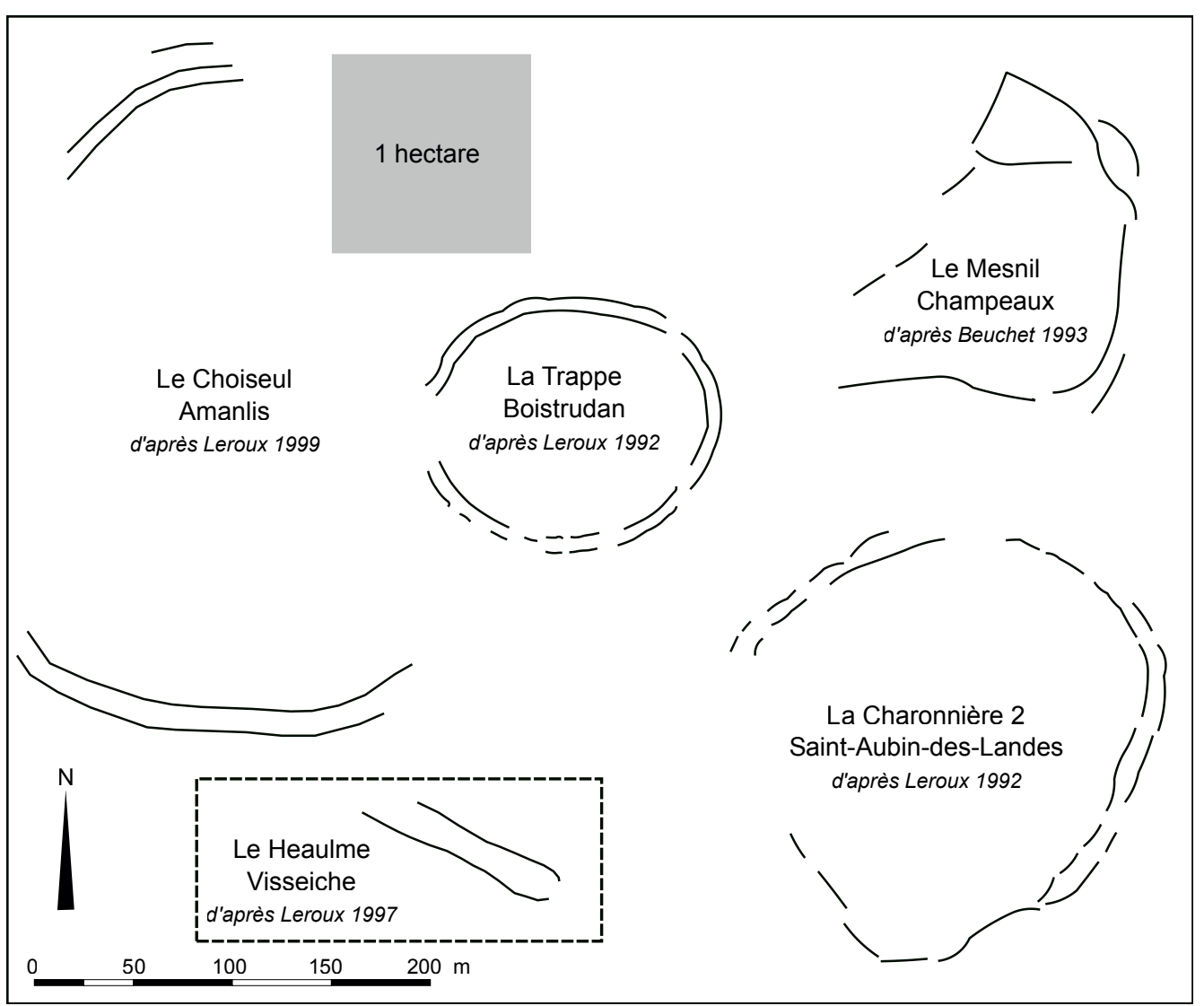

Figure 4 : Enceintes et possible sépulture type Passy observées en prospection aérienne par G. Leroux dans le secteur étudié.

Figure 4: Enclosures and possible Passy-type burial with aerial prospection by $G$. Leroux. 
associait un tertre et des menhirs, ce qui pourrait le rapprocher du tertre tumulaire de la Croix-Saint-Pierre à Saint-Just (35); Bézier, 1886; Briard et al., 1995).

\section{Les architectures de pierres dressées}

Avant tout, nous rappellerons ici la longue chronologie des pierres dressées, dont certaines le sont au sein d'un alignement dès le milieu du V $V^{e}$ millénaire, comme le Grand Menhir Brisé de Locmariaquer (56) et d'autres toujours dans un alignement au début du III millénaire comme au Gâvres (44; Cassen, 2009; Cassen et al., 2013).

Les menhirs seuls sont au nombre de dix-sept, dont un douteux (tabl. 1). Autrement, on recense sept gisements témoignant d'architectures plus complexes, dont la plupart ont été détruites (Balazé, Dourdain, La Bouëxière, 35) ou déplacées (La Rimbergère à Janzé, 35). Si deux alignements sont connus (Le Theil de Bretagne, La Métaierie Neuve à Janzé, 35), le plus important est celui du Bois de la Mancellière à La Bouëxière (35) avec 23 blocs sur $700 \mathrm{~m}$ de longueur, sur lequel vient se greffer au moins une ligne de 12 blocs s'étalant sur $100 \mathrm{~m}$ de long (Gaudin et al., 2000).

\section{Les lames de haches ou d'herminettes découvertes isolément}

Des lames de haches de dimensions variées se rencontrent sur le territoire; nous les utiliserons ici en sachant pertinemment que ces objets ne signalent pas forcément un gisement et qu'ils ont pu être conservés sur la très longue durée. Par contre, leur grande variété typologique et leurs matériaux donnent des informations sur l'insertion du secteur étudié au sein des réseaux d'échange.

Les lames de haches en roches vertes communes dominent en nombre. On notera quelques exemplaires supérieurs à $10 \mathrm{~cm}$ de longueur. À Saint-Didier (35), la Tizonnais, une grande lame polie en roche verte de $320 \mathrm{~mm}$ a été découverte mais a disparu de son lieu de conservation (Provost, 1989). La grande lame en dolérite de Bréal-sous-Vitré (35) de $234 \mathrm{~mm}$ (fig. 5, no 1 ) présente un profil irrégulier et une forme arquée avec un flanc rectiligne et une grande plage égrisée, où le bouchardage se perçoit encore très bien; C.-T. Le Roux l'a regroupé avec quelques autres exemplaires, dont le matériau viendrait des Côtesd'Armor mais pas de Plussulien (notes d'analyse conservées au Laboratoire de l'UMR 6566). L'autre exemplaire de

\begin{tabular}{|c|c|c|c|c|c|c|c|}
\hline Commune & Nom & Commentaire & $\begin{array}{l}\text { Hauteur } \\
\text { en cm }\end{array}$ & Matériau & Bibliographie & Vallée & $\begin{array}{c}\text { CODE } \\
\text { PROSP, } \\
\text { N,10,0 }\end{array}$ \\
\hline Brie & Belle-Épine & Menhir détruit & 415 & Schiste rouge & $\begin{array}{c}\text { Bézier, 1883; Briard et al., } \\
2004\end{array}$ & La Seiche & 1 \\
\hline Bruz & Pierre de Cahot & Menhir & 390 & Schiste rouge & $\begin{array}{c}\text { Bézier, 1883; Collin, } \\
\text { 1932; Briard et al., } 2004\end{array}$ & La Seiche & 1 \\
\hline $\begin{array}{l}\text { Chartres- } \\
\text { de-Bretagne }\end{array}$ & Le Bourg & Menhir détruit & 180 & Quartzite & $\begin{array}{c}\text { Bézier, } 1883 \text {; Briard et al., } \\
2004\end{array}$ & La Seiche & 1 \\
\hline Janzé & $\begin{array}{l}\text { La Métaierie } \\
\text { Neuve }\end{array}$ & $\begin{array}{l}\text { Alignement de } \\
3 \text { blocs }\end{array}$ & 0 & Schiste rouge & Briard et al., 2004 & La Seiche & 0 \\
\hline Janzé & La Rimbergère & $\begin{array}{l}\text { Alignement de } \\
6 \text { blocs détruits }\end{array}$ & 0 & Schiste rouge & $\begin{array}{c}\text { Bézier, 1883; Briard et al., } \\
2004\end{array}$ & La Seiche & 0 \\
\hline Janzé & Les Bouillons & $\begin{array}{c}\text { Menhir } \\
\text { (déplacé de son } \\
\text { lieu d'origine) }\end{array}$ & 184 & Schiste rouge & $\begin{array}{c}\text { Bézier, 1886; Briard et al., } \\
2004\end{array}$ & La Seiche & 1 \\
\hline $\begin{array}{l}\text { Le Theil-de- } \\
\text { Bretagne }\end{array}$ & Forêt du Theil & $\begin{array}{l}\text { Alignement de } \\
5 \text { blocs }\end{array}$ & 0 & $\begin{array}{l}\text { Schiste rouge et } \\
\text { grès armoricain }\end{array}$ & Corre, 2016 & La Seiche & 3 \\
\hline Retiers & $\begin{array}{l}\text { Le Pierre de } \\
\text { Richebourg }\end{array}$ & Menhir & 315 & Quartzite & $\begin{array}{l}\text { Bézier, 1883; Bézier, } \\
\text { 1886; Collin, 1932; } \\
\text { Briard et al., } 2004\end{array}$ & La Seiche & 3 \\
\hline Retiers & Les Jeusseries & Menhir détruit & 120 & Non renseigné & $\begin{array}{c}\text { Bézier, 1886; Briard et al., } \\
2004\end{array}$ & La Seiche & 1 \\
\hline Retiers & Pierrelet & Menhir détruit & 180 & Schiste rouge & $\begin{array}{l}\text { Bézier, 1883; Collin, } \\
\text { 1932; Briard et al., } 2004\end{array}$ & La Seiche & 1 \\
\hline $\begin{array}{l}\text { Saint-Er- } \\
\text { blon }\end{array}$ & Telle & Menhir détruit & 400 & $\begin{array}{l}\text { Quartzite } \\
\text { noirâtre }\end{array}$ & $\begin{array}{c}\text { Bézier, 1883; Briard et al., } \\
2004\end{array}$ & La Seiche & 0 \\
\hline Saulnières & $\begin{array}{l}\text { La Jouaudière / } \\
\text { La Table aux Fées }\end{array}$ & $\begin{array}{l}\text { Menhir couché } \\
\text { et débité }\end{array}$ & 450 & Schiste rouge & $\begin{array}{c}\text { Bézier, 1883; Briard et al., } \\
2004\end{array}$ & La Seiche & 1 \\
\hline
\end{tabular}




\begin{tabular}{|c|c|c|c|c|c|c|c|}
\hline $\begin{array}{l}\text { Argentré- } \\
\text { du-Plessis }\end{array}$ & Crespel & $\begin{array}{l}\text { Menhir } \\
\text { douteux }\end{array}$ & 240 & Quartz blanc & $\begin{array}{c}\text { Meuret, 1993;Trautmann } \\
\text { et al., } 2011\end{array}$ & La Vilaine & 3 \\
\hline Balazé & La Hardelière & $\begin{array}{l}\text { Groupe de } \\
4 \text { menhirs } \\
\text { détruits }\end{array}$ & 0 & Quartzite & $\begin{array}{l}\text { Bézier, 1883; Collin, } \\
\text { 1932; Briard et al., } 2004\end{array}$ & La Vilaine & 0 \\
\hline Champeaux & La Haute Pierre & Menhir & 407 & $\begin{array}{l}\text { Grès de Saint- } \\
\text { Germain-sur- } \\
\text { Ille }\end{array}$ & $\begin{array}{l}\text { Bézier, 1883; Collin, } \\
\text { 1932; Briard et al., } 2004\end{array}$ & La Vilaine & 3 \\
\hline Cornillé & La Baudonnière & $\begin{array}{c}\text { Menhir couché } \\
\text { et débité }\end{array}$ & 0 & $\begin{array}{l}\text { Microgranite } \\
\text { gris }\end{array}$ & Trautmann et al., 2011 & La Vilaine & 3 \\
\hline Dourdain & $\begin{array}{l}\text { La Bédouhan- } \\
\text { nerie }\end{array}$ & $\begin{array}{l}\text { Plusieurs men- } \\
\text { hirs détruits au } \\
\text { XIX }^{\text {e }} \text {. }\end{array}$ & 0 & Non renseigné & $\begin{array}{c}\text { Bézier 1883; Briard et al. } \\
2004\end{array}$ & La Vilaine & 3 \\
\hline $\begin{array}{l}\text { La } \\
\text { Bouëxière }\end{array}$ & La Mancellière & $\begin{array}{l}\text { Alignement } \\
\text { complexe }\end{array}$ & 0 & $\begin{array}{l}\text { Grès de Saint- } \\
\text { Germain-sur- } \\
\text { Ille et quartz } \\
\text { blanc laiteux }\end{array}$ & $\begin{array}{c}\text { Collin, 1934; Briard et al., } \\
2004\end{array}$ & La Vilaine & 3 \\
\hline $\begin{array}{l}\text { La } \\
\text { Bouëxière }\end{array}$ & $\begin{array}{l}\text { La Plardais-La } \\
\text { Salmonais }\end{array}$ & $\begin{array}{l}\text { Trois menhirs, } \\
\text { dont un détruit }\end{array}$ & 0 & Non renseigné & $\begin{array}{l}\text { Bézier, 1883; Collin, } \\
\text { 1934; Gaudin et al., } \\
\text { 2000; Briard et al., } 2004\end{array}$ & La Vilaine & 3 \\
\hline Landavran & Clairet & Menhir & 390 & Quartzite gris & $\begin{array}{c}\text { Bézier, 1883; Collin, } \\
\text { 1932; Briard et al., } 2004\end{array}$ & La Vilaine & 3 \\
\hline Landavran & Le Bas Landavran & Menhir & 250 & $\begin{array}{l}\text { Quartz-arénite } \\
\text { de la Lande- } \\
\text { Murée }\end{array}$ & $\begin{array}{l}\text { Inédit. Informatrice : } \\
\text { A. Paquet }\end{array}$ & La Vilaine & 3 \\
\hline $\begin{array}{l}\text { Livré-sur- } \\
\text { Changeon }\end{array}$ & La Roche Piquée & Menhir & 385 & $\begin{array}{l}\text { Quartzite (grès } \\
\text { armoricain) }\end{array}$ & $\begin{array}{c}\text { Bézier, 1883; Collin, } \\
\text { 1932; Briard et al., } 2004\end{array}$ & La Vilaine & 3 \\
\hline $\begin{array}{l}\text { Pocé-les- } \\
\text { Bois }\end{array}$ & La Pierre Blanche & Menhir & 372 & $\begin{array}{l}\text { Grès de Saint- } \\
\text { Germain-sur- } \\
\text { Ille }\end{array}$ & Briard et al., 2004 & La Vilaine & 0 \\
\hline $\begin{array}{l}\text { Pocé-les- } \\
\text { Bois }\end{array}$ & Villaumur & $\begin{array}{c}\text { Menhir } \\
\text { (déplacé de son } \\
\text { lieu d'origine) }\end{array}$ & 194 & $\begin{array}{l}\text { Grès de Saint- } \\
\text { Germain-sur- } \\
\text { Ille }\end{array}$ & $\begin{array}{l}\text { Bézier, 1883; Collin, } \\
\text { 1932; Briard et al., } 2004\end{array}$ & La Vilaine & 3 \\
\hline
\end{tabular}

Tableau 1 : Architectures de pierres dressées des vallées de la Seiche et de la Vilaine.

Table 1: Menhirs in the Seiche and Vilaine valleys.

Bréal-sous-Vitré (35) en proviendrait toutefois (fig. 5, no 4). Une lame de hache à bouton en dolérite a été découverte à Coultru à Juvigné (53), non loin des sources de la Vilaine (Kerdivel, 2019a; fig. 5, n 2). À Moulins (35), la lame de hache en dolérite se distingue par son côté massif et épais (fig. 5, n 3). À Janzé (35), celle de la Gare est atypique par sa forme et présente une surface bouchardée ou altérée. La lame mince montre qu'il s'agit d'une dolérite (fig. 5, $\mathrm{n}^{\circ} 5$ ). D'autres lames de haches supérieures à $10 \mathrm{~cm}$ de longueur sont connues à Vern-sur-Seiche (35) et Châtillonen-Vendelais (35; fig. 5, $\mathrm{n}^{\text {os }} 6$ et 7).

Il existe aussi un cortège important de lames en dolérite, dont la longueur est inférieure à $10 \mathrm{~cm}$, comme à Amanlis (35; fig. 6, $\mathrm{n}^{\circ} 4$ ). Celle de Dompierre-du-Chemin (35; fig. $\left.6, n^{\circ} 1\right)$ présente quelques stries sur une de ses faces et des irrégularités dans le polissage, marquant sans ambiguïté son remploi en pierre à affûter de nos campagnes, ce dont L. Marsille témoigne en évoquant son voisin, «sabotier de son état, donne à ses outils le dernier fil sur un très beau celtae en diorite qu'il ne donnerait à aucun prix, vu son utilité " (Marsille, 1911). C'est le même cas pour la lame de Chelun (35; fig. $7, \mathrm{n}^{\circ}$ 8). Beaucoup montrent des traces d'utilisation, comme celles de Louvigné-de-Bais (35; fig. 6, $\mathrm{n}^{\circ}$ 5) ou de Bais (35; fig. 6, $\mathrm{n}^{\circ} 2$ ). D'autres semblent nettement avoir été retaillées et polies comme celle de Juvigné ( 53 ; fig. $6, \mathrm{n}^{\circ} 3$ ) ou de Chelun (35; fig. 7 , $\mathrm{n}^{\circ} 7$ ). La lame de Rennes (35) s'avère intéressante car elle n'a pas été terminée : elle ne présente pas de tranchant et ses bords ne sont que bouchardés (fig. $6, \mathrm{n}^{\circ} 6$ ). D'autres ne se retrouvent qu'à l'état de fragment (Vern-sur-Seiche et Champeaux, 35; fig. 6, nos 7 et 8). Une lame de hache en dolérite à bords équarris a été retrouvée dans une fosse gauloise de la ZAC de la Grande Haie à Vitré (35), mais elle n'a pas été retrouvée par nos soins (Hamon, 2010). 
Pour ce type de haches à bords équarris en roche verte, nous avons proposé par ailleurs pour le polissage l'emploi d'une machine plutôt réservée aux lames en silex (Kerdivel, 2019b; Pelegrin, 2012).

Les lames de haches en fibrolite sont plus rares : l'exemplaire de Marcillé-Robert (35) pourrait provenir de l'atelier de Plouguin (29) et correspondrait aux exemplaires fréquents du Néolithique moyen (Pailler, 2009; fig. 7 , $\mathrm{n}^{\circ} 3$ ). Une autre lame dans ce matériau a été découverte à la Pucelais à Amanlis (35) mais n'a pas pu être retrouvée en 2019 (Provost, 1989). À Bais (35), J.-C. Meuret a signalé une lame en jadéite, que nous n’avons pas pu retrouver en 2019.

D'autres matériaux moins fréquents et donc ayant moins attiré l'attention des chercheurs sont plus difficiles à déterminer. À Janzé (35), deux lames courtes ont été recueillies par un particulier dans son jardin. Si le la roche verte litée de l'une n'a pu être identifiée plus précisément (fig. 7, $\mathrm{n}^{\circ} 1$ ), l'autre est blanchâtre à grisâtre et renvoie à des exemplaires que l'on rencontre sporadiquement en Mayenne et qui évoque une roche volcanique, de type ignimbrite (fig. 7, nº 2). À Villiers-Charlemagne (53), au sud des vol- canites carboniferes d'Entrammes justement, plusieurs collections de surface attesteraient d'une possible production locale (coll. B. Bodinier, GRAM). À Marcillé-Robert (35), une hache en roche siliceuse est conservée au musée de Bretagne : elle mériterait d'être déterminée (fig. 7, no ${ }^{\circ}$ ). À Chelun (35), plusieurs lames de formes variées ont fait l'objet de lames minces, qui ont montré à C.-T. Le Roux (fiches conservées au Laboratoire de Rennes 1 et transcrites par C.-T. Le Roux) l'emploi de différents grès : grès à ciment plus ou moins recristallisé (fig. $7, \mathrm{n}^{\circ} 5$ ), grès micacé à ciment siliceux recristallisé (fig. $7, \mathrm{n}^{\circ} 6$ ), grès à ciment pélitique (fig. $7, \mathrm{n}^{\circ}$ 9), voire de pélites sédimentaires (fig. 7 , $\mathrm{n}^{\circ} 7$ ). De toutes, une retient l'attention par la présence des négatifs de mise en forme non effacés par le polissage (fig. $7, n^{\circ} 5$ ). Pour finir, une préforme taillée dans un quartzite, pièce exceptionnelle, a été signalée à Champeaux (35) mais n’a pas pu être retrouvée en 2019 (Beuchet, 1993).

Les lames en silex sont tout aussi rares. On en trouve quelques exemplaires sur le site du Rallion 1 à La Bouëxière (35; Onnée et al., 2000), de la Maison Neuve à Argentré-du-Plessis (35; Meuret, 1986) et à Amanlis au lieu-dit Laval (35; Giot, 1958).
Figure 5 : Lames de haches en roches vertes communes (dolérite ?) entières de plus de $10 \mathrm{~cm}$ de longueur.

Figure 5: Stone axes in "common green stone" (dolerite?) longer than $10 \mathrm{~cm}$.

1 et 4 : Bréal-sous-Vitré (35), 2 : Coultru à Juvigné (53), 3 : Villechien à Moulins (35), 5 : La Gare à Janzé (35), 6 : Bel Air à Vern-sur-Seiche, 7 : La Morlière à Châtillon-en-Vendelais (35). Les haches mentionnant MdR sont conservées au Musée de Rennes. La hache de Juvigné est conservée au Musée d'Ernée.

1 and 4: Bréal-sous-Vitré (35), 2: Coultru à Juvigné (53), 3: Villechien à Moulins (35), 5: La Gare à Janzé (35), 6: Bel Air à Vern-sur-Seiche, 7: La Morlière à Châtillon-en-Vendelais (35). The ones labeled MdR are kept at the Museum of Rennes. The stone axe from Juvigné is in the Museum of Ernée.

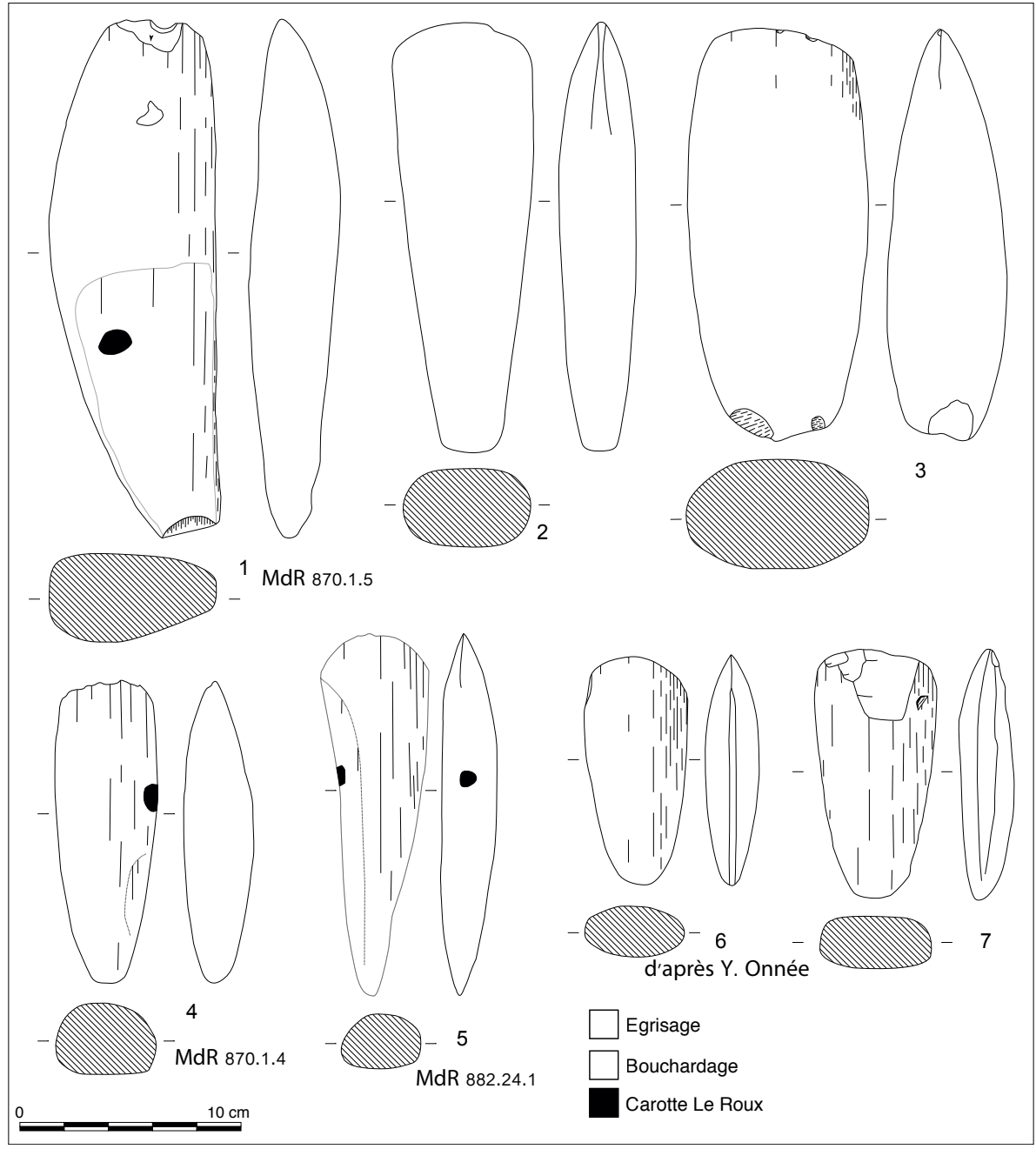




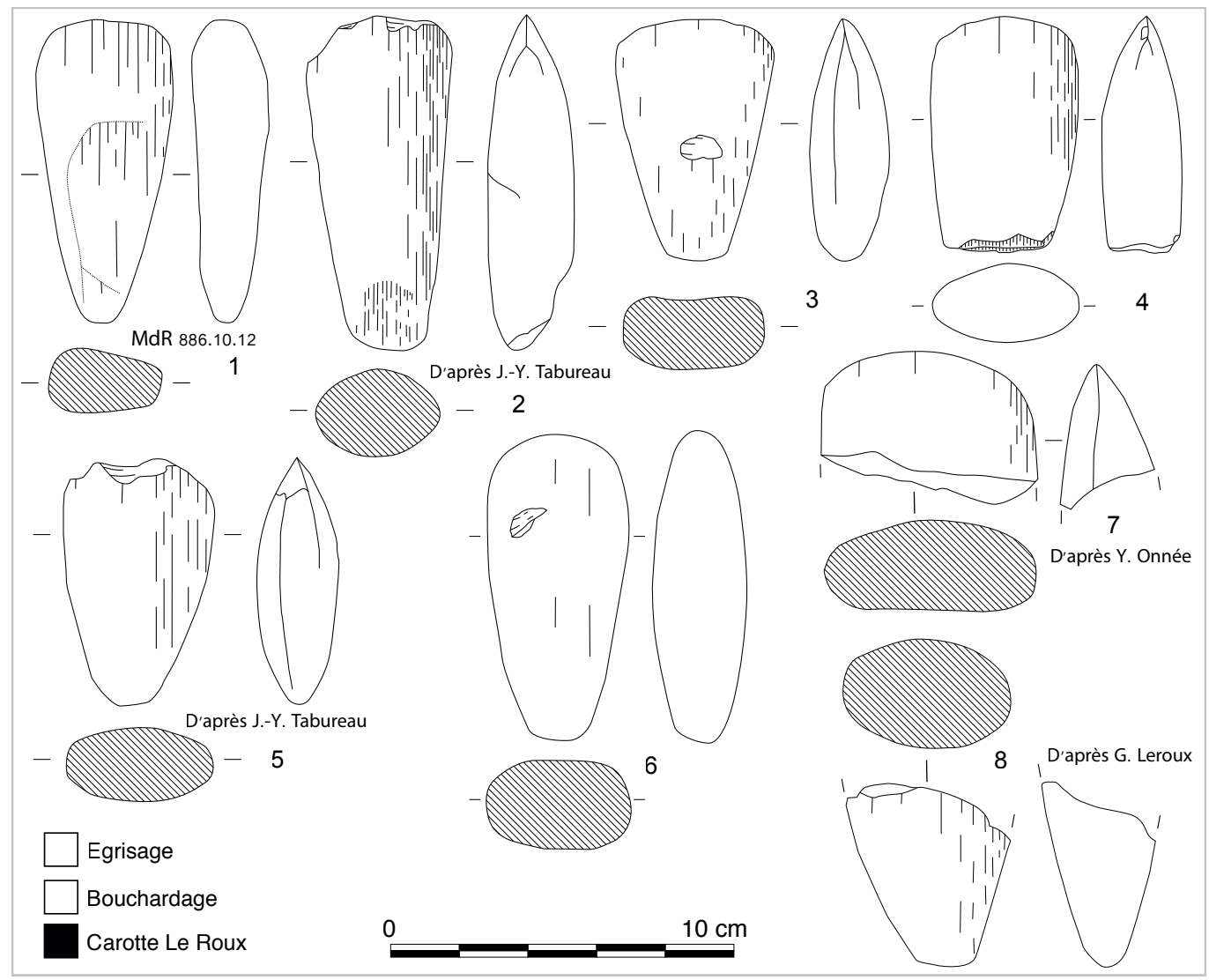

Figure 6 : Lames de haches en roches vertes communes (dolérite ?) entières de moins de $10 \mathrm{~cm}$ de longueur et autres fragments.

Figure 6: Stone axes in "common green stone" (dolerite?) of less than $10 \mathrm{~cm}$ in length and fragments.

1: Dompierre-du-Chemin, 2 : Les Landes d’Elberte à Bais (35), 3: La Daligaudais à Juvigné (53), 4 : Le Pâtis-Loyer à Amanlis (35), 5 : Le Bois-d'Yà Louvigné-de-Bais (35), 6: Rue de Nantes à Rennes (35), 7 : Gaudon à Vern-sur-Seiche (35), 8: Champeaux (35). Les haches mentionnant MdR sont conservées au Musée de Rennes. La hache de Juvigné est conservée au Musée d'Ernée. La hache de Rennes est conservée au Laboratoire d’Anthropologie à Rennes.

1: Dompierre-du-Chemin, 2: Les Landes d'Elberte à Bais (35), 3: La Daligaudais à Juvigné (53), 4: Le Pâtis-Loyer à Amanlis (35), 5: Le Bois-d'Yà Louvigné-de-Bais (35), 6: Rue de Nantes à Rennes (35), 7: Gaudon à Vern-sur-Seiche (35), 8: Champeaux (35). The examples labeled MdR mention are kept in the Museum of Rennes. The stone axe from Juvigné is in the Museum of Ernée. The stone axe from Rennes is from Rennes's Anthropological Laboratory.

\section{L'OCCUPATION DE CE SECTEUR D'ILLE-ET-VILAINE AU COURS DU NÉOLITHIQUE}

particuliers sur la longue durée ou au cours d'opérations préventives ou de sauvetage.

$28,5 \%$ des gisements ont été découverts par l'inventaire (signalements anciens précédant la recherche systématique postérieure à la seconde guerre mondiale), quand un peu plus de 20,5\% l'ont été par la prospection, essentiellement pédestre (16,5\%). Sur ce dernier mode de découverte, il faut observer que plus de gisements ont été découverts ainsi dans la vallée de la Vilaine que dans celle de la Seiche, situation inverse pour la prospection aérienne (3 dans la vallée de la Seiche et 2 dans la vallée de la Vilaine). Un tel résultat est d'autant plus étonnant que les terrains prospectables sont plus nombreux dans la vallée de la Seiche que dans la vallée de la Vilaine. Dans cette dernière d'ailleurs, on distingue nettement, au nordest, une vaste zone où les terrains sont non prospectables et n'ont été touchés par aucune opération préventive (le fameux domaine nord-armoricain). 


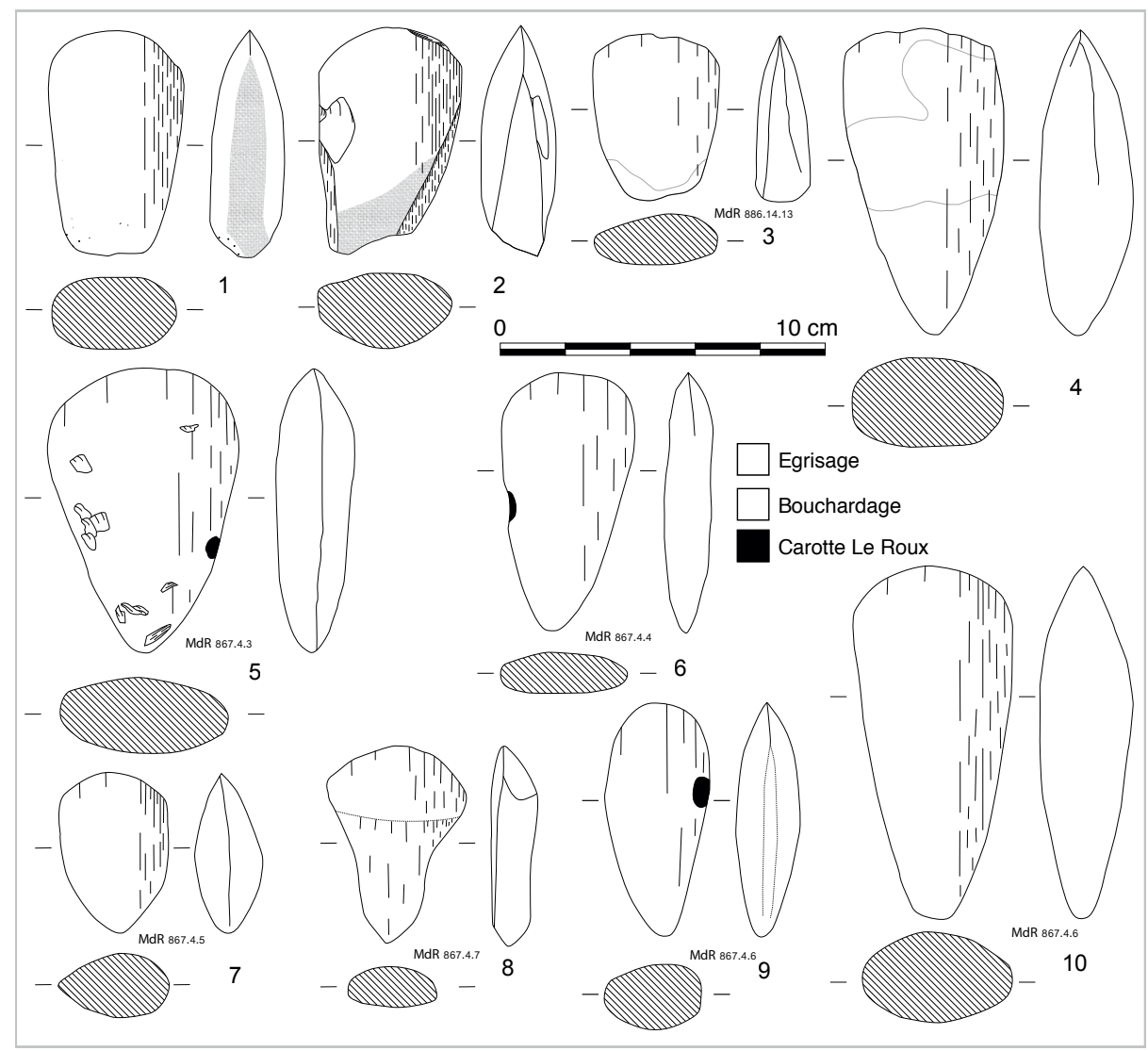

Figure 7 : Haches en matières autres.

Figure 7: Stone axes in other materials.

1 et 2 : 50, avenue du Général de Langle de Cary à Janzé (35) ; 3 et 4 : Marcillé-Robert (35) ; 5 à 10 : Chelun (35). 1 : Roche foliée indéterminée. 2 : Ignimbrite ? ; 3 : Fibrolite ; 4: Quartzite ? ; 5 à 10 : Grès divers. Les haches mentionnant MdR sont conservées au Musée de Rennes.

1 and 2: 50, avenue du Général de Langle de Cary à Janzé (35); 3 and 4: Marcillé-Robert (35); 5 to 10: Chelun (35). 1: Indeterminate foliated rock. 2: Ignimbrite?; 3: Fibrolite; 4: Quartzite?; 5 à 10: Various sandstones. The objects labeled MdR are in the Museum of Rennes.

Le dynamisme économique de Vitré Communauté et de Rennes Métropole, sans compter la ligne à grande vitesse, ont conduit à de nombreuses opérations d'archéologie préventive (fig. 8). On peut d'ores et déjà noter qu'il y a eu proportionnellement plus d'opérations archéologiques et de superficies observées ${ }^{1}$ dans la vallée du Blosne $(4,7 \%)$ que dans la haute vallée de la Vilaine $(1,3 \%)$ et que dans celle de la Seiche $(2,3 \%)$. Cela est dû tout simplement à l'activité économique de ce secteur. (À ce propos, tous les gisements y ont d'ailleurs été découverts au cours d'une opération préventive sur des terrains

1. Les opérations archéologiques ont été appréhendées au moyen de la couche de données mises en ligne sur le site https:/geobretagne.fr/geonetwork/srv/fre/catalog.search\#/metadata/51587d33-febb-4bef-adfe-bc673d06b26d. Les pourcentages indiqués ne sont que des valeurs maximales puisque dans le cas des diagnostics, toute la superficie du projet est prise en compte alors que ce sont des tranchées qui ont été réalisées en pratique. II faut par ailleurs ajouter que diagnostic et tranchée ne veulent pas dire que le fait préhistorique ait été toujours appréhendé : cela dépend aussi de la compétence du responsable d'opération et de ses équipiers, sujet tabou dans la profession... prospectables ou pouvant permettre la prospection ${ }^{2}$ et l'abondance de zones artificialisées suppose une urbanisation importante). Les deux autres vallées montrent un contraste important avec une vallée de la Seiche plus explorée en préventif que celle de la Vilaine, ce qui se ressent dans le nombre de gisements découverts au cours de ces opérations, respectivement 14 et 6 .

Ces observations préalables induisent une certaine précaution dans l'interprétation de nos données. Ainsi, l'impact un peu plus important de l'archéologie préventive dans la vallée de la Seiche et la plus forte abondance de zones non prospectables dans la vallée de la Vilaine (forêt de Chevré, de la Corbière et différents bois) impliquent une dichotomie entre les vallées dans la répartition des

2. Les catégories de prospectabilité ont été définies dans Kerdivel, 2012, p. 27-28. L'échelle de production de CORINE Land Cover, la couche d'information ayant servi à cette caractérisation au 1/100 000 et la plus petite unité cartographiée de 25 ha, sont trop imprécises pour une analyse à l'échelon local. Cela n’a pas permis de nuancer la couche « terrains pouvant permettre la prospection ». 


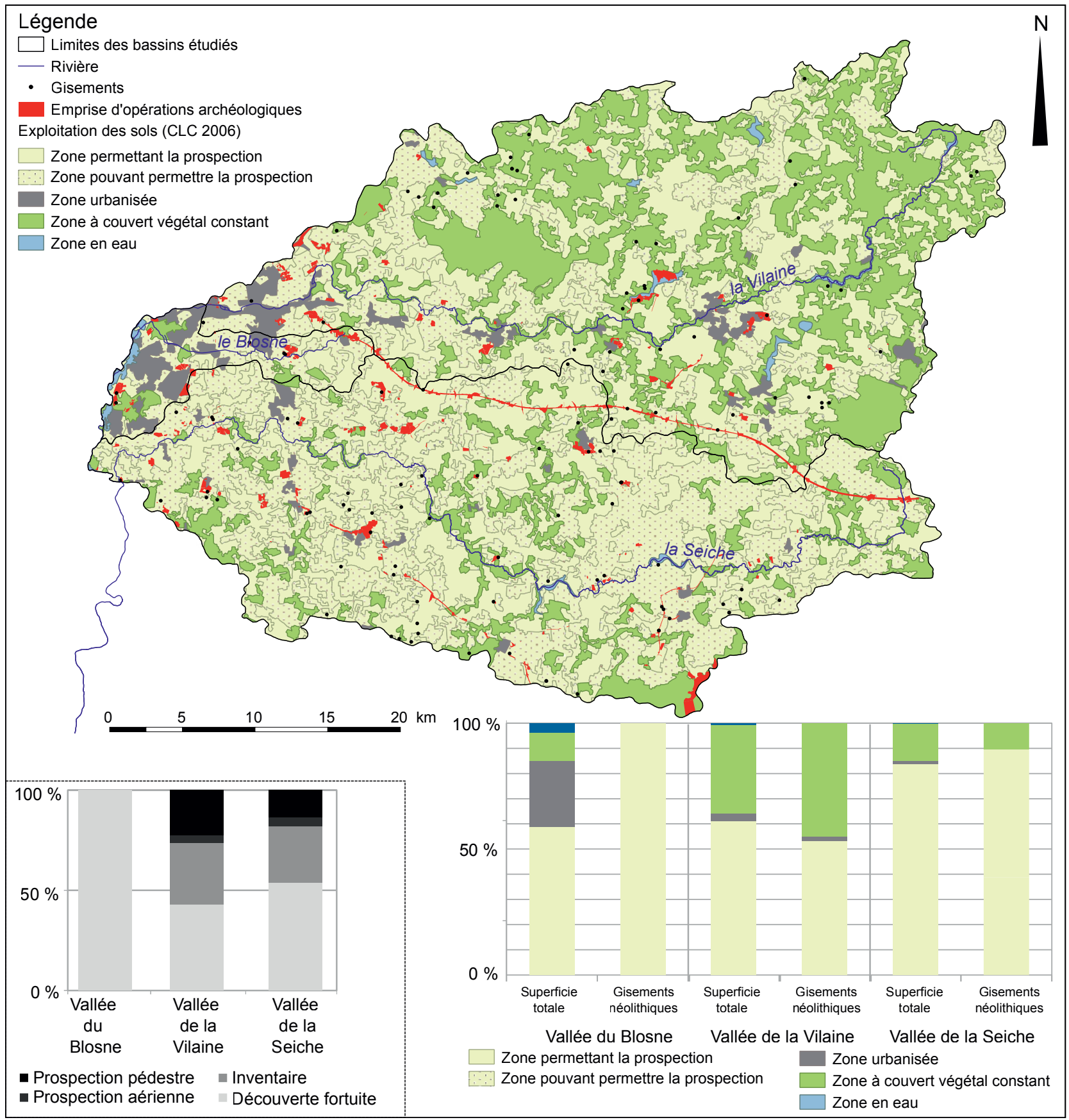

Figure 8: Carte des terrains selon leur prospectabilité et surfaces des opérations archéologiques.

Figure 8: Map showing fields according to their prospectability and the surface area of archaeological works.

L'histogramme de gauche présente les modalités de découvertes par vallée et celui de droite compare pour chaque vallée les superficies selon leur prospectabilité et les gisements selon la prospectabilité du terrain sur lequel il a été découvert.

The histogram on the left shows the discoveries made in each valley and the one on the right compares, for each valley, the areas according to their prospectability and the sites in relation to the prospectability of the field in which they were discovered.

gisements et une hétérogénéité dans les études qui ont pu être menées. Toutefois, on peut aussi voir qu'aux gisements découverts en prospection dans la vallée de la Vilaine plus nombreux répondent ceux découverts fortui-
Par ailleurs, il faut ajouter que le nord de Vitré reste la zone la moins touchée par des travaux archéologiques quels qu'ils soient, ce qui entame fortement notre analyse spatiale. 


\section{Les gisements et le substrat géologique}

Le déterminisme environnemental et géologique en particulier est très contesté tant par les géographes que par les archéologues, dans le sillage d'un raisonnement qui relève de l'ontologie matérialiste selon la définition de P. Descola (Descola, 2005, p. 241-280). Malgré cette contestation, il nous semble important d'examiner le rapport entre les deux, ne serait-ce que pour les mégalithes qui sont de facto tributaires de ce paramètre.

\section{Approches chronologiques (fig. 9)}

Au Néolithique ancien, les trois gisements ne se rencontrent que sur le Briovérien centre-armoricain. Cela va perdurer avec le Néolithique moyen, où les gisements investissent durablement ce même substrat. Il faut constater d'ailleurs, comme nous l'avions observé pour les enceintes, qu'il s'agit d'un substrat fluide, c'est-à-dire facile à creuser (Kerdivel, 2014). Les grès et autres roches plus ou moins indurées, datées entre l'Ordovicien et le Silurien, sont peu occupés, si l'on excepte le cas du Bertry à La Bouëxière (35). C'est d'ailleurs dans ce secteur que plusieurs gisements s'installent au Néolithique récent et final. Ainsi, si l'occupation du domaine briovérien centre-armoricain se renforce là encore, il semble que les populations néolithiques investissent d'autres substrats, notamment le nord-est de notre secteur. Bien que les gisements datés y soient peu nombreux, nous rappellerons la découverte à Châtillon-en-Vendelais (35) d'une hachemarteau, par exemple (De la Grancière et Harmois, 1916).

\section{Le cas particulier de la Roche aux Fées renforcé}

La Roche aux Fées à Essé (35) est donc ce monument emblématique bien connu et maintes fois décrit (Bézier, 1883). Il s'agit d'un dolmen angevin, c'est-à-dire à portique (L'Helgouac'h, 1965). "Toute la structure mégalithique est réalisée en blocs de schiste pourpré ordovicien » provenant des affleurements de la forêt du Theil (35), à $5 \mathrm{~km}$ au sud à vol d'oiseau (Le Roux, 1998). D'ailleurs, ces blocs ont récemment fait l'objet d'une étude géomorphologique, venant affiner leur provenance probable (Sellier, 2017). L'emploi de blocs de provenance si lointaine « montre combien le lieu d'installation devait s'imposer avec force aux bâtisseurs, même si les raisons nous en échappent " (Le Roux, 1998). En effet, l'emplacement topographique précis du monument (ni le point le plus élevé du secteur, ni le plus bas) comme son orientation dans l'axe du lever du soleil au solstice d'hiver (Meuret, 1986) aurait aussi bien pu être obtenu ailleurs. Or, la force de ce choix d'implantation est renforcé par la répartition générale des gisements du Néolithique moyen que nous observons, où l'on voit que le substrat briovérien a un attrait particulier pour les Néolithiques : tous les gisements de cette phase s'y trouvent. Comment expliquer alors cet attrait du briovérien, qui ne semble pas n'être qu'un biais?

\section{Les mégalithes: déplacements de matériaux ou constructions sur place}

Outre le cas de la Roche aux Fées, plusieurs monuments mégalithiques, même non datés, présentent une distribution étonnante au regard de leur matériau de construction (fig. 2). Ainsi, les dolmens ou assimilés se retrouvent uniquement associés à l'Ordovicien. C'est le cas, au nord, avec le dolmen de l'Écotais ou, au sud, avec les mégalithes de Pierrelet, des Noyers et du Plessix. Le fait que ces monuments aient été détruits ou non retrouvés par nos soins en est tout à fait regrettable : une observation attentive des éléments architecturaux les composant aurait pu nourrir notre réflexion.

L'inféodation de certaines pierres dressées à ce même Ordovicien se retrouve dans le sud de notre secteur de manière caricaturale. Ainsi, l'alignement des gisements qui suit le substrat ordovicien a déjà fait couler beaucoup d'encre. Ainsi, J.-C. Meuret fait remarquer qu'ils se répartissaient pour une bonne part le long du chemin des Saulniers, connu dans le canton de Retiers (35; Meuret, 1986). Il est à noter que ces monuments, quand ils n'ont pas été détruits, sont dans leur majorité en schiste pourpré et dressés sur le substrat qui a fourni le matériau... Point de déplacement spectaculaire ici comme nous l'avons vu pour la Roche aux Fées.

Par contre, au nord et à l'ouest de Vitré, des cas de déplacements de blocs avant érection sont à souligner (fig. 10). Ainsi, le bloc débité, probable menhir, de la Baudonnière à Cornillé (35) est en microgranite mais installé sur un affleurement de schiste (Trautmann et al., 2011). Il est en danger au moment d'écrire ces lignes, situé en bordure de carrière. Les deux menhirs de Villaumur à Pocé-lesBois (35) et de la Haute Pierre à Champeaux (35) sont tous deux en grès de Saint-Germain-sur-Ille, mais dressés sur la formation d'Andouillé, composée de siltites noires et d'argilites : rien qui puisse fournir de tels blocs, et encore moins de plus de $4 \mathrm{~m}$ (Trautmann et al., 2011). Il faut aller chercher du côté des roches du groupe de la Bouëxière qui affleurent à quelques centaines de $\mathrm{m}$ pour trouver le matériau nécessaire à ces blocs. C'est le même matériau pour la Pierre Blanche à Pocé-les-Bois (35), dressée sur le briovérien tendre. Là encore, le choix de l'emplacement importait plus que les efforts pour déplacer le matériau. Plus au nord, le menhir inédit du Bas Landavran à Landavran (35), en grès de la Lande-Murée, a nécessité un transport de quelques centaines de mètres alors que son 


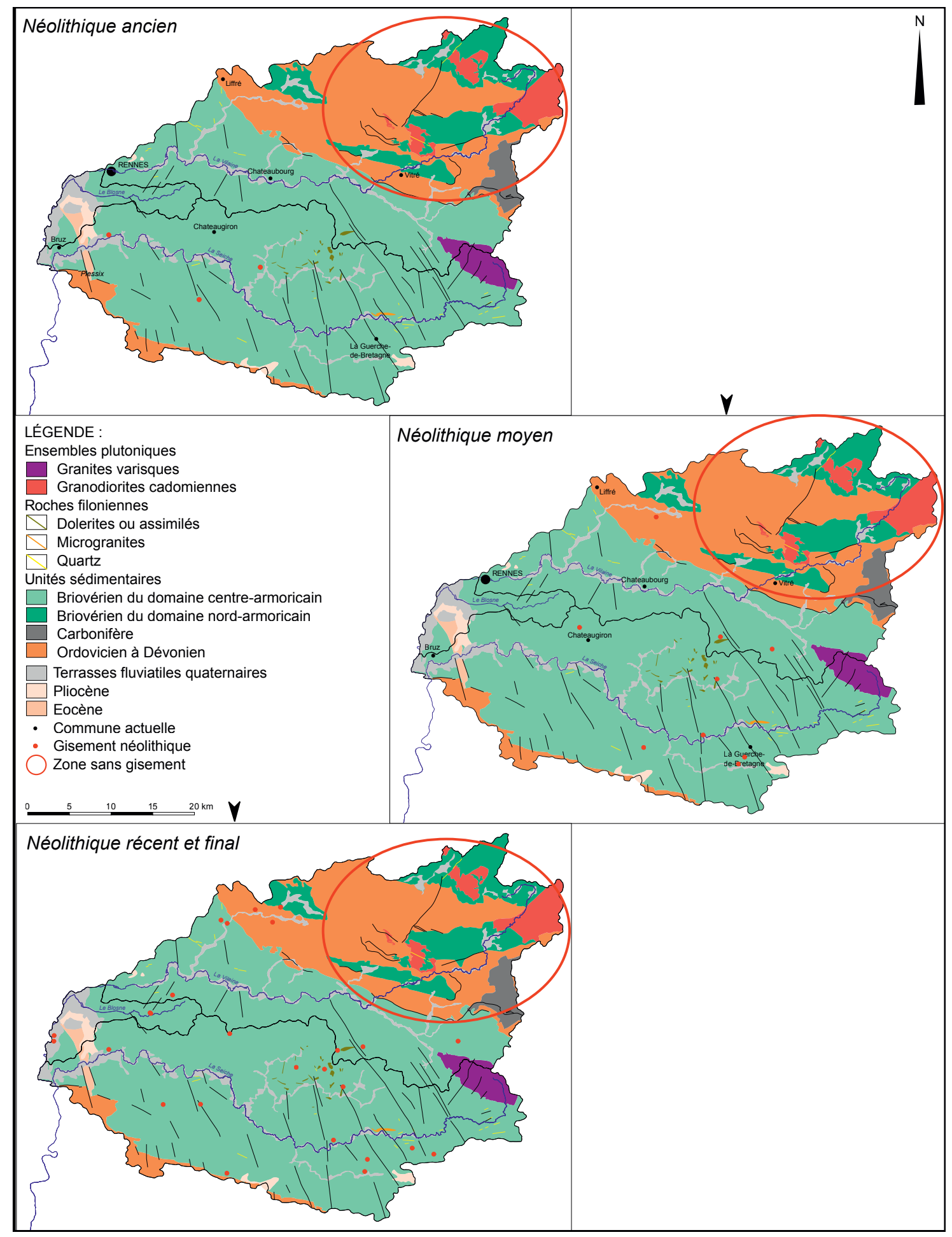

Figure 9: Carte géologique montrant la répartition des gisements en fonction de leur datation.

Figure 9: Geological map showing Neolithic settlements.

homologue du Clairet sur la même commune a probablement été dressé sur le lieu d'affleurement de la roche.

Le même phénomène de déplacement a été observé pour plusieurs blocs de l'alignement du Bois de la p. 105) ou pour l'ensemble détruit de la Hardelière à Balazé (35; Collin, 1932).

Au sein des mégalithes conservés et observables, on constate deux groupes se distinguant par le choix de l'emplacement et du matériau de construction. Dans 
Figure 10 : Carte géologique simplifiée du secteur de Vitré. Les pierres dressées sont colorées selon la matière qu'ils emploient.

Figure 10: Geological map (simplified) of the Vitré area. The menhirs are coloured according to the type of stone.

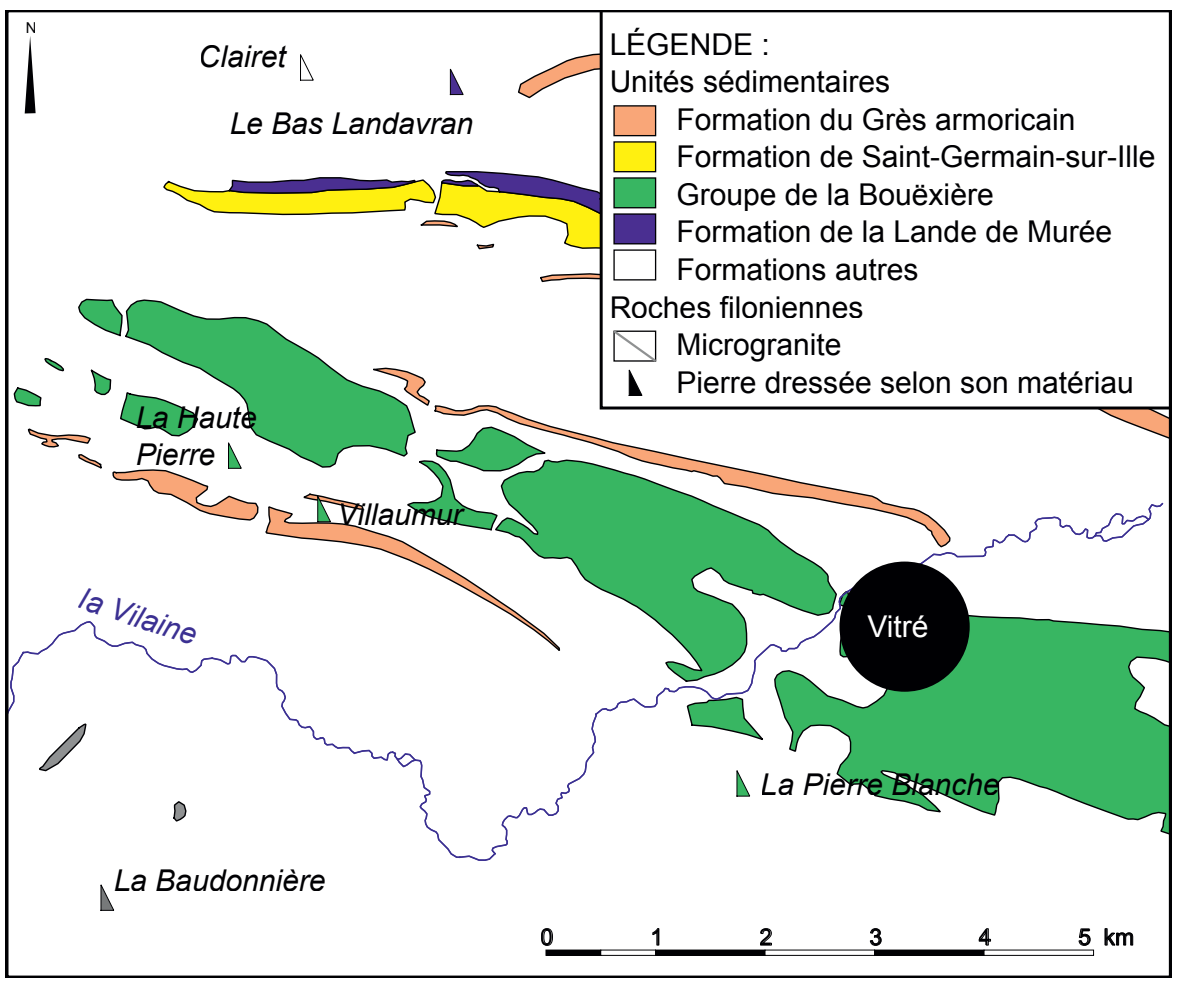

un groupe, c'est bien l'emplacement qui prévaut, leur constructeur va chercher ailleurs le matériau mégalithique. Dans l'autre, le matériau est immédiatement disponible dans l'environnement de construction. Tout en gardant à l'esprit que les pierres dressées ont pu être recyclées ou déplacées dès le Néolithique jusqu'à nos jours, on peut se demander si le choix de l'emplacement a vraiment la même influence dans les deux cas.

\section{Les gisements dans leur situation topographique respective}

Comme nous l'avons écrit plus haut, la géologie influence une topographie et un paysage monotone pour lesquels il semble difficile de déceler des spécificités d'implantation des gisements néolithiques. Ces implantations ont été appréhendées manuellement selon une méthode déjà développée (Kerdivel, 2012, p. 28-29). Si l'on regarde l'ensemble des gisements par phase et pour quelques-uns d'entre eux par type (fig. 11), on constate une forte préférence de la position médiane au cours du Néolithique ancien. À l'inverse, la position haute va être préférée au Néolithique moyen, suivie de la basse alors que la position médiane va être délaissée. Par la suite, au Néolithique récent et final, l'implantation dans le paysage se fait de manière plus homogène avec une préférence pour la position médiane et basse. La situation haute s'avère délaissée.

$\mathrm{Au}$ Néolithique moyen, les sites funéraires sont aux deux extrêmes : celui de la Roche aux Fées est en position haute quand le possible enclos funéraire de Visseiche (35) est en position basse. Les trois gisements qui se rapprochent le plus d'un habitat se situent eux en position haute : Domloup (35) et les deux sites de Rannée (35).

Au Néolithique récent et final, des deux sites funéraires recensés, seule la sépulture campaniforme des Rimbaudières appelle un commentaire grâce à sa localisation exacte : elle est en position dominante, ce qui se retrouve par ailleurs sur les marges du Massif armoricain (Kerdivel, 2012). Pour les sites non funéraires, on constate que les deux bâtiments d'Ossé (35) et de Bais (35) - dans l'ordre chronologique que suggèrent les datations ${ }^{14} \mathrm{C}-$ sont en position haute. La Pinelière à La Guerche (35) se distingue par sa situation médiane, quand le site des Rives du Blosne à Chantepie (35) est lui plutôt en position basse. Les indices de site avec structures archéologiques renvoient à une situation topographique comparable : celle d'une occupation homogène de la topographie.

Parmi les gisements non datés, les sites ceinturés méritent d'être analysés ici tant ils sont associés classiquement mais souvent excessivement aux dernières étapes du Néolithique. Ainsi, trois d'entre elles se situent en position dominant le paysage, soit en éperon, soit en rebord de plateau. Seule l'enceinte de Champeaux (35) est installée en position médiane sur un versant de vallée.

Des monuments mégalithiques non datés, les deux dolmens de Retiers (35) et de La Chapelle-Erbrée (35) semblent avoir été en position haute quand ceux d'Orgères (35) ont plutôt été en position basse. Mais ce sont 


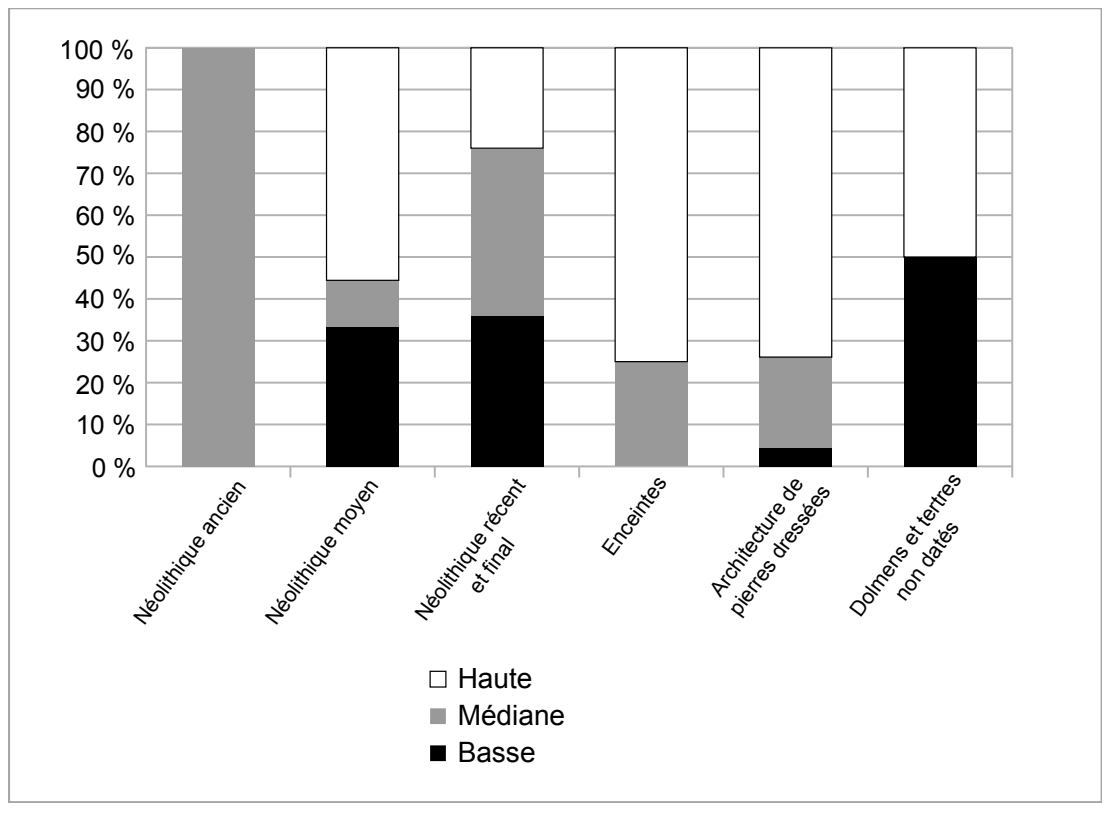

Figure 11 : Histogramme présentant la position topographique des gisements par période chronologique et de quelques gisements particuliers non datés.

Figure 11: Bar graph of the topographical layout of Neolithic and undated sites (enclosures, menhirs, dolmens). les architectures de pierres dressées qui appellent les commentaires les plus pertinents. Ainsi, il faut noter que la position haute est grandement préférée dans dix-sept sur vingt-trois cas. Les plus longs alignements observables tendent à courir le long de la pente comme au bois de la Mancellière à La Bouëxière (35), les plus courts coupent les plateaux où ils sont installés.

\section{Les gisements et la variabilité des sols actuels}

Discuter de la qualité des sols au Néolithique est une tâche ardue voire impossible tant ceux-ci ont dû évoluer depuis cette période et les modifications du paysage et des pratiques agraires qui ont suivi. Toutefois, nous considérons qu'il est possible d'appréhender quelques tendances, ce que nous avions soutenu et démontré dans un autre travail (Kerdivel, 2012, p. 172-179). C'est ce que nous nous proposons de faire ici : dégager quelques tendances dans une dynamique chronologique ${ }^{3}$ (fig. 12). Pour ce faire, nous avons repris le concept d'indice d'exploitabilité des sols que nous avions proposé (Kerdivel, 2012, p. 37-38).

Pour mémoire, l'indice de 1 à 4 montre un gradient depuis des terrains viables (1) à d'autres qui montrent au

3. Les sols ont été appréhendés grâce à la carte des sols de Bretagne disponible à cette adresse: http://www.sols-de-bretagne.fr/ et grâce à celle de Mayenne que nous a transmis Marie-Christine Puntello (CD 53). Nous avons ensuite traité les données suivant la méthode mise en place pour notre thèse et que n'avions appliqué qu'à la région Poitou-Charentes (Kerdivel, 2012, p. 172-178). Pour ce faire, nous avons utilisé la table attributaire de la carte des sols. L'acidité a été estimée par rapport au substrat, l'hydromorphie a été simplifiée en trois classes (absente, plus ou moins présente, forte), la texture estimée selon le triangle de Jamagne l'a été selon le triangle du GEPPA en tâchant de suivre les trois classes de notre thèse (terres franches, sols séchants/ moins deux critères limitants (4). Sur l'ensemble du secteur étudié, l'indice 2 domine fortement et se concentre dans la vallée de la Seiche et au sud de la vallée de la Vilaine. L'indice 3 se retrouve à cheval entre les vallées de la Seiche de la Vilaine puis ponctuellement dans ces deux vallées. Celle de la Vilaine concentre les zones d'indice 4. Seules quelques zones de la vallée de la Vilaine ainsi qu'une vaste zone au sud de Rennes à cheval sur Bruz et Chartres-de-Bretagne (35) présentent un indice 1, c'est-à-dire des sols favorables. Comme pour le substrat géologique, une certaine dualité du secteur est marquée entre un tiers septentrional et les deux tiers restants.

Les gisements datés dont nous avons observé la distribution selon cet indice sont les sites non funéraires, les indices de site avec structures et le matériel de surface. De manière générale, on constate une tendance à aller d'une occupation de quelques types de sols spécifiques au Néolithique ancien vers celles de tous les types de sols au Néolithique récent et final, à tel point que le diagramme en pourcentage de la superficie et de celui du NRF sont très semblables. Le Néolithique moyen s'avère un peu particulier avec un gisement en indice 3 et un en indice 4. Ce dernier s'avère être le ramassage du Bertry à La Bouëxière (35) pour lequel nous avons déjà émis quelques réserves quant à sa datation. Quant à l'autre, il s'agit du site de la Sallerie à Rannée (35).

\section{Gisements et chemins anciens}

Cela fait maintenant bien longtemps que l'on ne discute plus la relation entre les mégalithes et les voies romaines ou autres chemins anciens (Curtet, 1959). Cela est probablement dû à l'écart chronologique entre les deux périodes concernées et la difficulté, dans le cas des chemins, de les 


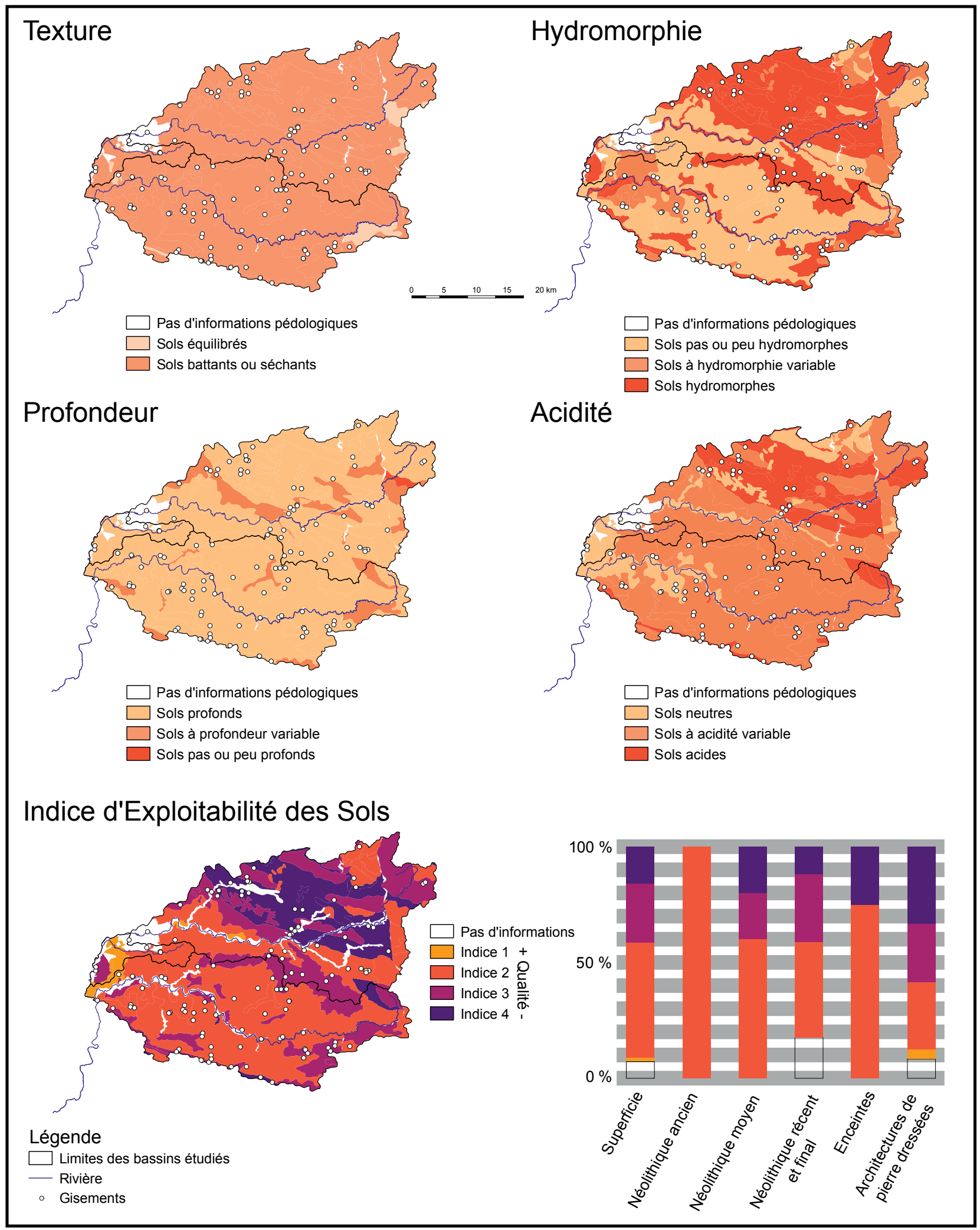

Figure 12 : Carte des sols actuels selon différents critères (texture, hydromorphie, profondeur, acidité et Indice d'exploitabilité du sol ou IES). Figure 12: Map of soils according to texture, hydromorphy, depth, acidity and Soil Index Exploitability.

L'histogramme à droite compare les superficies selon leur IES, les gisements par période chronologique et de quelques gisements particuliers non datés. The bar graph on the right compares the areas according to Soil Index Exploitability, the sites by chronological period and undated sites. 
dater. Tâche donc difficile mais pas impossible : le chemin le plus ancien connu dans notre secteur est parallèle à la D 777 et est daté de l'âge du Bronze (Leroux, 2015). En l'état, on ne connaît pas de chemin néolithique hors site d'habitat. Quelques auteurs ont toutefois proposé des cheminements hypothétiques qu'il reste à trouver (Berthaud et al., 2015).

L'emploi des Systèmes d'informations géographiques donne des outils d'analyse et facilite la discussion de l'éventuelle relation entre cheminements et gisements préhistoriques, c'est en tout cas le parti qu'ont pris d'autres auteurs que nous (Fairén Jiménez et al., 2006).

J.-C. Meuret a, nous l'avons rappelé, signalé la proximité de certains menhirs avec le «chemin des Sauniers". Il nous a paru pertinent de réexaminer la véracité de ce lien spatial. Pour ce faire, nous avons confronté les chemins anciens avec l'ensemble des gisements ${ }^{4}$.

Ainsi, sur l'ensemble des chemins que nous avons pu cartographier (fig. 13), seul le chemin des Sauniers (3C) présente des pierres dressées à moins de $500 \mathrm{~m}$ de son tracé (idem à moins d'1 km). On notera alors que si ce n'est le dolmen de Pierrelet et son menhir associé, aucun des mégalithes funéraires n'est concerné par une quelconque proximité avec un chemin ancien ni même les autres architectures de pierres dressées, ailleurs qu'au sud du secteur étudié.

Par contre, quelques rares gisements se trouvent concernés par la proximité avec un chemin ancien : c'est le cas de ceux de Piré-sur-Seiche (croisement 3E - 1H), du Blosne à Chantepie ou de Visseiche $(1 \mathrm{H})$. Les quelques autres gisements concernés sont de petites collections de surfaces, dont deux de la fin du Néolithique (Liffré et La Bouëxière) voire des objets isolés (haches). Il semble s'agir là plus d'un hasard que d'une réalité préhistorique.

\section{LE PEU QUE L'ON PEUT AVANCER DE L'OCCU- PATION DE L'ESPACE ET DE LA GESTION DES RES- SOURCES AU COURS DU NÉOLITHIQUE DANS LES VALLÉES DE LA SEICHE ET DE LA HAUTE-VILAINE...}

Dans cet essai de synthèse sur ce secteur de la Bretagne, nous nous efforcerons d'écrire une histoire dynamique de l'occupation de l'espace et de la gestion des ressources au cours de ces 3000 ans que dure le Néolithique, mais aussi de montrer en quoi nos données s'inscrivent dans les problématiques les plus actuelles de la discipline.

4. La couche d'informations géographiques sur les chemins anciens a été construite à partir des données du site internet de Philippe Saint-Marc [http:// voiesromaines35.e-monsite.com/pages/les-voies-introduction-1.html] et consiste en polylignes tracées à partir des Scan25 de l'IGN, en employant la nomenclature du site Internet.

\section{Les débuts du Néolithique}

Les débuts du Néolithique dans ce secteur sont donc à attribuer à la culture du Villeneuve-Saint-Germain désormais bien connue en Bretagne depuis les découvertes des années 1990 de Saint-Étienne-en-Coglès (35), situés immédiatement au nord de notre secteur, jusqu'à celles des années 2010, dont Pen Hoat Salaun à Pleuven (29) (Hinguant et al., 1998; Nicolas et al., 2013). La synthèse la plus récente des données nous a été proposée dans un article auquel il convient d'ajouter les découvertes les plus récentes, y compris celle largement inédite de Kervouric à Lannion (22; Pailler et al., 2008).

Dans le cas qui nous occupe, il faut d'abord constater qu'il s'agit dans deux cas sur trois de tessons découverts hors contexte. À Janzé (35), l'hypothèse d'un habitat en amont a été formulée. À Piré-sur-Seiche (35), bien que le gisement soit structuré, l'organisation et l'arasement des structures ne permettent guère de discuter la nature de l'occupation du site, mais il n'est pas sans rappeler le site de Bazouges-Château-Gontier (53; Valais et al., 2008). Nous avions écrit que l'occupation de l'espace au VSG n'était pas seulement le fait des maisons trapézoïdales si classiques, mais pouvait peut-être prendre des formes qu'il reste à caractériser : occupation légère pour une fréquentation courte (Kerdivel, 2012, p. 185) ? Nous avions souligné aussi l'inféodation à des environnements favorables. Or, tous ces gisements sont découverts uniquement sur le briovérien et sur des sols qui, s'ils ne sont pas les meilleurs, ne sont pas non plus les pires. Tous situés dans la vallée de la Seiche, nous pourrions facilement affirmer que cette rivière et sa vallée sont le moteur de la néolithisation de notre secteur. Or, c'est sans compter sur le fait qu'il s'agit de la vallée tout simplement la plus propice aux découvertes et qui a fait l'objet du plus de surfaces fouillées.

\section{Le plein Néolithique moyen}

Le basculement vers le Néolithique moyen se fait doucement via la culture Cerny, dont un gisement est connu dans le secteur à Domloup (35). Plus troublante est la découverte en prospection aérienne à Visseiche de traces qui renvoient aux sépultures de type Passy, largement inconnues sur le Massif armoricain, mais bien connues sur ses marges (Kerdivel, 2012, p. 275). Nous tenons là un élément de première importance qu'il conviendra de valider ou d'invalider dans le futur.

À partir de $4300 \mathrm{BCE}$, les gisements se multiplient avec cette fois-ci, des natures d'occupation un peu plus perceptibles. Ainsi à la Sallerie à Rannée (35), les données disponibles renvoient aux fosses-silos connues par exemple à Grentheville ou Banville (14) (Kerdivel et Hamon, 2010; 


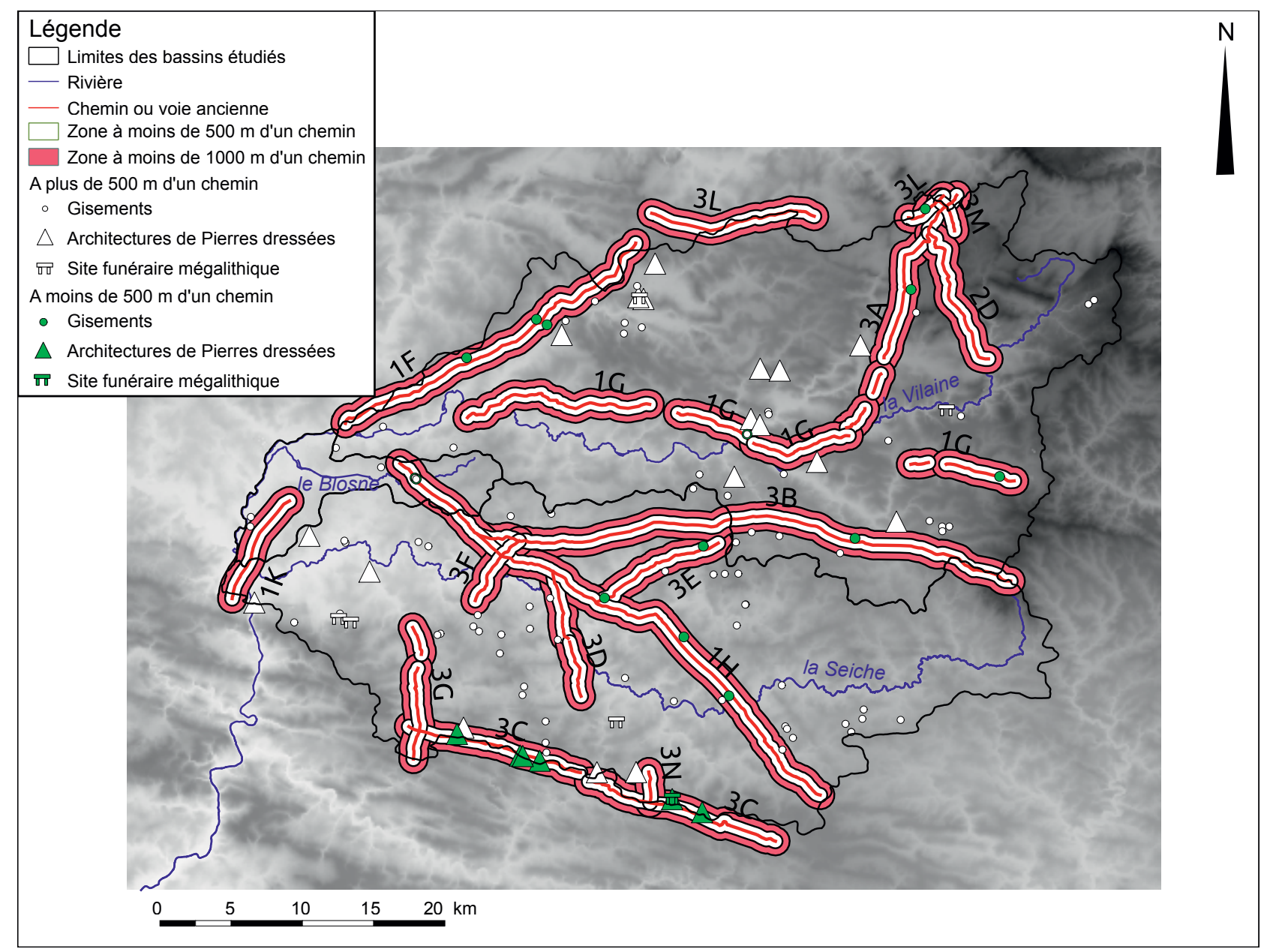

Figure 13 : Carte topographique et analytique des chemins anciens répertoriés et des monuments mégalithiques dans le secteur étudié.

Figure 13: Topographical and analytical map of ancient roads with known megalithic monuments in the study area.

Chancerel et al., 2006). Cela en fait d'ailleurs un gisement particulier dans notre secteur. Toutefois, le mobilier y est pauvre. À proximité de Rannée, un ensemble de foyers en cuvette signale là encore une occupation domestique. Ces gisements implantés en position haute font écho à la situation topographique du dolmen de la Roche aux Fées à Essé (35). Comme pour la phase précédente, le substrat briovérien et la vallée de la Seiche sont occupés, d'une manière peut-être préférentielle qu'il faudra confirmer. Une incursion sur les hauteurs ordoviciennes pourrait être le site du Bertry à La Bouëxière (35), dont la datation reste toutefois discutable. Indiscutable est par contre l'implantation du site de la Sallerie dans un environnement pédologiquement plutôt défavorable (terrain hydromorphe), mais là encore, la prudence est de rigueur : l'hydromorphie a pu beaucoup varier depuis le Néolithique.

Ce n'est pas surprenant, au cours de cette phase où l'approvisionnement en matières premières lithiques lointaines (silex normand ou angevin) continue selon des réseaux de diffusion mis en place dès le Villeneuve Saint-
Germain, comme en atteste le mobilier de Bais (35), que le seul monument mégalithique daté de cette phase soit construit avec des blocs provenant justement de plusieurs kilomètres. Si ces modalités d'approvisionnement lointaines ne sont pas systématiques au cours du Néolithique moyen (il suffit de regarder du côté des monuments contemporains normands), nous y voyons là toutefois un élément significatif. Ainsi, les quelques pierres dressées du secteur de Vitré (35) qui ont fait l'objet pour certaines d'un déplacement de plusieurs centaines de mètres seraient à dater de cette phase du Néolithique, où l'anthropisation de l'espace se fait de plus en plus prégnante. Cette hypothèse reste toutefois à étayer par des fouilles ou des datations directes, par thermoluminescence peut-être (Cassen et al., 2013).

Si la production de lames de hache en roche du socle primaire débute dès le Néolithique moyen, comme l'attestent l'exploitation de dolérite de Plussulien (22) ou plus près encore (dans le bassin versant à l'est du secteur étudié), celle de Saint-Germain-le-Guillaume (53), aucun 
élément n'en atteste dans ces deux vallées au cours de cette phase, malgré la présence de filons doléritiques dans le secteur de Bais (35; Le Roux, 1999; Kerdivel et al., 2017). L'abondance et la diversité des lames de hache recensées démontrent que ces deux vallées sont bien insérées dans les réseaux de diffusion. L'existence aux confins des sources de la Vilaine d'une hache à bouton en parfait état, production apparemment typique de Plussulien mais non datée, prend une place toute symbolique. Une éventuelle typochronologie des haches en dolérite permettra peut-être prochainement de préciser nos données, notamment d'un point de vue chronologique (thèse en cours de L. Bénéteaud).

Ces données ne viennent pas infirmer ce que nous écrivions au sujet de cette phase en proposant, sur la base des six formes de sédentarité proposées par A. Whittle, une mobilité forte au sein d'un territoire anthropisé et marqué par le mégalithisme et un monumentalisme funéraire imposant; bien au contraire, elles semblent les renforcer (Whittle, 2001; Kerdivel, 2012, p. 189-190). C. Scarre n'avait pas proposé autre chose dans son analyse de toute la période Néolithique pour la Bretagne: "The twin keys to understanding the demography of Neolithic Brittany are mobility and fluctuation" (Scarre, 2001, p. 301).

\section{Le Néolithique récent, final et le Chalcolithique}

Cette phase, ou plus exactement ces phases encore aujourd'hui en recherche d'une définition plus précise dans l'Ouest de la France, montrent une nette augmentation du nombre de gisements. Cela est dû dans notre secteur à une meilleure identification du mobilier de cette phase mais cela renvoie plus globalement à une croissance de la population comme on l'observe aux marges du Massif armoricain (Kerdivel, 2012, p. 195-196) ou ailleurs (Caraglio, 2020).

Cela induit une plus grande variété de gisements. On connaît ainsi deux bâtiments : un du Néolithique récent à Ossé (35) et l'autre du Néolithique final à Bais (35). Seule une allée couverte très probable est connue à La Bouëxière (35). Les indices de site avec structures archéologiques sont aussi plus nombreux, comme les mobiliers de surface ou les pièces isolées. Par exemple, les fragments de poignards pressigniens attestent sans surprise de l'insertion de ce secteur dans les réseaux d'échanges de ces produits depuis l'actuelle Touraine (Ihuel, 2012). De même, les haches-marteaux témoignent d'indices de gisements datés de cette phase du Néolithique (Barbier, 1992). Si les informations relatives à l'origine des matières premières ne sont pas disponibles pour nos gisements, faute d'études, il faut souligner que le seul site d'extraction et de fabrication de lames de hache est daté de cette phase aux Rives du Blosne à Chantepie (35). L'ampleur et la méthode d'exploitation n'ont malheureusement pas été suffisamment étudiées. On sait toutefois qu'il s'agit de l'exploitation d'un microgranite (Blanchet, 2004). Or, cette roche n'a été que peu employée sur le Massif armoricain pour façonner des haches, seules deux haches sont connues dans l'article de référence de Cogné et Giot (1952). On peut alors vraisemblablement émettre l'hypothèse que la multiplication des types de matières premières employées dans l'industrie lithique et bien connues à la fin du Néolithique s'applique aussi aux lames de haches et que différentes matières vont faire l'objet d'une exploitation (Ghesquière et Guyodo, 2008) : ce pourrait être le cas du cortège des grès, observé pour les lames de Chelun (35) ou de l'ébauche possible de Champeaux (35).

La croissance de la population se perçoit aussi avec une implantation des gisements sur l'ensemble de l'environnement : les substrats ordoviciens sont clairement investis dans le secteur de La Bouëxière, les sols plus défavorables aussi (IES 3 et 4), ainsi que tous les types de positions topographiques.

Nous avons proposé plus haut, à titre d'hypothèse, que certaines pierres dressées ayant fait l'objet de déplacement de plusieurs centaines de mètres dans le Pays de Vitré pourraient être datées du Néolithique moyen. A contrario, les pierres dressées situées à moins de $500 \mathrm{~m}$ du chemin des Sauniers forment pour cela un cas particulier déjà observé (Meuret, 1986; 1993). La plupart sont dressées avec les matériaux locaux sans guère de déplacement majeur. Or, on sait que des pierres sont dressées depuis le milieu du $\mathrm{V}^{\mathrm{e}}$ millénaire jusqu'à l'âge du Bronze, et parfois l'âge du Fer, attestant d'une longue durée de cette pratique (Laporte et al., 2015). On sait aussi qu'un chemin situé à Piré-sur-Seiche a pu être daté de l'âge du Bronze et que c'est de cette période que datent les plus anciens parcellaires connus dans l'Ouest à Tatihou (Marcigny et Ghesquière, 2003; Leroux, 2015). En Irlande, dans les marais de Behy et Glenulra (comté de Mayo), d'autres sont même datés du Néolithique final (O'Kelly, 2001, p. 65-67). On peut donc formuler l'hypothèse de pierres dressées tardives de l'âge du Bronze voire au mieux du Néolithique final balisant un chemin, tout en gardant à l'esprit que ce phénomène a été aussi observé à Lillemer (35), le long d'un chemin en bois daté du Néolithique moyen (Laporte et al., 2007). Or, parcellaires et chemins sont à lier à une structuration politique de l'espace comme l'a justement rappelé James C. Scott (Scott, 2019).

Un tel schéma renvoie à des modalités d'occupation de l'espace différentes des phases précédentes et plutôt ici du type d'une « sédentarité courte avec stabilité résiden- 
tielle d'une année sur l'autre " pour reprendre les termes d'A. Whittle (2001; Kerdivel, 2012, p. 192). Le tout dans un territoire qui s'anthropise plus encore qu'auparavant. Cela semble d'ailleurs différer de ce que l'on connaît à la fin du IV et au début du III millénaire av. n. è. dans les sites lacustres, où il semble s'agir plutôt d'une « sédentarité ancrée avec continuité résidentielle génération après génération" (Whitle, 2001; Scarre, 2001, p. 304-305); schéma que l'on peut retrouver peut-être en aval de la vallée de la Vilaine (Tinévez, 2004).

\section{POUR L'IMMENSITÉ DE CE QU'IL NOUS RESTE À DÉCOUVRIR DANS CE SECTEUR}

En guise de conclusion, nous aimerions jeter les bases d'une programmation de la recherche sur le Néolithique de ce secteur, où l'on constate que dans une zone de taille réduite toutes les problématiques actuelles du Néolithique trouvent un écho (CNRA, 2016) : "Achèvement des processus de néolithisation, Plein-Néolithique/ Chalcolithique et formation de l'âge du Bronze, construire des chroniques précises et fiables, systèmes techniques, archéologie funéraire et anthropologie des populations $\mathrm{du}$ passé et territorialité, écologie et régimes de mobilité. » Il n'est donc point besoin de s'éloigner géographiquement pour tenter de combler les lacunes dans notre connaissance de cette période!

$\mathrm{Si}$, dans les lignes qui précèdent, nous avons fait un inventaire et l'avons confronté à quelques critères environnementaux pour ensuite le placer dans une dynamique chronologique afin d'en établir une synthèse, force est de constater qu'il s'agissait plutôt d'un bilan de ce que l'on ne sait pas.

Il nous reste donc dans les prochaines années à renforcer l'inventaire de l'existant en retrouvant certaines données disparues et en tâchant de collecter des données encore inconnues. Cela passera probablement par une prospection pédestre des territoires environnants et un inlassable travail de vulgarisation auprès des habitants actuels des vallées de la Vilaine et de la Seiche.

Sur cette trame générale, il nous semblerait pertinent que des travaux soient menés sur le VSG découvert à Janzé (35), des sondages au Heaulme à Visseiche (35) et à Pierrelet à Retiers (35) et qu'une étude de la carrière potentielle de Chantepie (35) soit menée. Il s'agit de travaux bien difficiles à mener pour nous actuellement, mais qui, nous en sommes certains, permettraient de mieux comprendre la Néolithisation, la complémentarité entre sépultures ou territoires (Essé-Retiers) et la variabilité des roches exploitées pour les lames de hache. Tout un programme!

\section{Remerciements}

Nous souhaitons remercier les bénévoles qui nous ont aidé à acquérir de l'information ou qui nous ont donné accès à leurs objets : Jean-Yves Barbedore, Virginie, Margaux et Éric Bigot, Gilbert Chesnel, André Corre et l'ensemble du Cérapar, Danièle Davenel, Évelyne Dugrée, Jean-Marie Fesneau, Martine Garnier, Jean Lebouc, Pierre Magnan, Georges Marin, Aurélie Paquet, Michel Hubert, Laurent Reusse, Philippe Saint-Marc.

Nous voulons aussi remercier les quelques professionnels qui nous ont aussi apporté leur aide : Lucie Bénéteaud (UMR 6566), Loïc Gaudin (ArkéoMap), Laurent Aubry (Inrap), Gwenaëlle Hamon (musée de Carnac), Jean-Claude Meuret (UMR 6566), CharlesTanguy Le Roux (UMR 6566), Anne-Louise Hamon (Inrap), Gilles Leroux (Inrap), Laure Cadars (MCC), Véronique Gendrot (MCC), Sébastien Toron (Éveha), Sandra Sicard (Inrap), Stéphane Blanchet (Inrap), Manon Six (musée de Bretagne), Christian Walter (Agro Campus Rennes), Blandine Mercier (Agro Campus Rennes).

Imparfait et lacunaire, ce travail de longue haleine, débuté initialement en 2017, a été mené sur la durée en parallèle d'une activité de gérant de bar, particulièrement chronophage. Nous remercions nos proches qui comprennent la passion qui nous anime pour la préhistoire et pour cette discipline qu'est l'archéologie qui, décidément, ne sera jamais réductible au carcan de la professionnalisation quoi qu'on en dise maintenant (Aubin et al., 2018, p. 13) : merci donc à Ruti et à Glen. Nous continuons à rêver à une Préhistoire du Futur, telle que l'avait esquissé P. Gouletquer en 1979 (Gouletquer, 1979).

\section{Bibliographie}

Aubin G., Le Roux C.-T. et Marcigny C., 2018, « Archéologie et archéologues ", dans Aubin G., Le Roux C.-T. et Marcigny C. (dir.), Sur le terrain avec les archéologues. 30 ans de découvertes dans l'Ouest de la France, Rennes, Presses universitaires de Rennes, p. 13-19.

Aubry L., 2017, Bretagne. Ille-et-Vilaine. Communes de Janzé et Amanlis. Zone économique du Bois de Teillay, Tranche 3, Rennes, SRA Bretagne/Inrap, $72 \mathrm{p}$.

Aubry L., 2005, Bruz (35), Cicé. Tranche 2, Rennes, SRA Bretagne/Inrap, $85 \mathrm{p}$.

Aubry L., 2010, Domloup, Ille-et-Vilaine ZAC économique du Tertre. Tranches 1 et 2, Rennes, SRA Bretagne/Inrap, 58 p.

Barbeau S., 2014, Ille-et-Vilaine, La Guerche de Bretagne/Rannéel Drouges, $R D$ 178-Déviation, tranche 3, Rennes, SRA Bretagne/Inrap, $138 \mathrm{p}$.

Barbeau S., 2011, Noyal-sur-Vilaine, Domloup, Cesson-Sévigné, Ille-et-Vilaine, LGV Rennes-Le Mans, section 1. Occupations 
gauloises, antiques et médiévales : des pôles de développement, Rennes, SRA Bretagne/Inrap, 178 p.

BARBIER S., 1992, "Les lames de haches perforées sur le territoire français. Essai de classification morphologique et étude comparative avec des instruments datés ", Groupe Vendéen d'Études Préhistoriques, 28, p. 12-53.

Berthaud G., Mens E., Raux P., Jupin S., Le Jeune Y.et Berson B., 2015, "Découverte d'une stèle gravée sur le plateau de la Bretellière, Saint-Macaire-en-Mauges (Maineet-Loire, France) ", INORA, 71, p. 7-15.

Beuchet L., 1993, Barrage de Villaumur 1992-1993, Rennes, SRA Bretagne/Inrap, 85 p.

BÉzIER P., 1883, Inventaire des monuments mégalithiques du département d'Ille-et-Vilaine, Rennes, C. Catel, 280 p.

BÉzIER P., 1886, Inventaire des monuments mégalithiques du département d'Ille-et-Vilaine, supplément, Rennes, C. Catel, 137 p.

Blanchet S., 2004, Chantepie "Les Rives du Blosne " (35), Rennes, SRA Bretagne/Inrap, 137 p.

Blanchet S., Nicolas T. et Toron S., 2012, "Des constructions inédites à la transition néolithique final-Bronze ancien en Bretagne : premier bilan ", INTERNÉO 9, Actes de la journée d'information du 17 novembre 2012 (Paris), Paris, Association INTERNÉO et Société préhistorique française, p. 135-145.

Bouillon R., 1989, "La sépulture mégalithique à entrée latérale du Petit-Vieux-Sou à Brécé (Mayenne) ", Revue archéologique de l'Ouest, 6, p. 51-70.

Briard J., Gautier M. et Leroux G., 1995, Les mégalithes et les tumulus de Saint-Just (Ille-et-Vilaine), Paris, Comité des travaux historiques et scientifiques (CTHS), $174 \mathrm{p}$.

Briard J., Langouët L. et Onnée Y., 2004, Les mégalithes du département d'Ille-et-Vilaine, Rennes, Institut Culturel de Bretagne et Centre Régional d'Archéologie d'Alet, coll. " Patrimoine archéologique de Bretagne », 122 p.

Caraglio A., 2020, «Une approche des dynamiques d'implantation des habitats à la fin du Néolithique provençal », Bulletin de la Société préhistorique française, 117, 3, p. 461-500.

Cassen S. (dir.), 2009, Autour de la Table. Explorations archéologiques et discours savants sur des architectures néolithiques à Locmariaquer, Morbihan (Table des Marchands et Grand Menhir), Nantes, Laboratoire de recherches archéologiques/CNRS/université de Nantes, 918 p.

Cassen S., Blain S., Guibert P., Querré G. et Chaigneau C., 2013, «Les pierres dressées de la forêt du Gâvre (Loire-Atlantique) : nature et origine des matériaux, premiers éléments de chronologie $\left({ }^{14} \mathrm{C}\right.$, OSL) ", ArcheoSciences, Revue d'archéométrie, 37, p. 173-188.

Chancerel A., Marcigny C. et Ghesquière E. (dir.), 2006, Le plateau de Mondeville (Calvados) du Néolithique à l'âge du Bronze, Paris, Éditions de la Maison des sciences de l'homme, Document d'Archéologie française, 99, 205 p.

CNRA, 2016, Programmation nationale de la recherche archéo- logique, Paris, ministère de la Culture et de la Communication, $216 \mathrm{p}$.

Cogné J. et Giot P.-R., 1952, "Étude pétrographique des haches polies de Bretagne, I ", Bulletin de la Société préhistorique française, 49, 8, p. 388-395.

Collin L., 1932, "Quelques monuments mégalithiques de l'Est et du Centre de l'Ille-et-Vilaine ", Bulletin et mémoires de la Société archéologique du département d'Ille-et-Vilaine, 58, p. 11-40.

Corre A., 2010, Rapport annuel de prospection, SRA Bretagne - CERAPAR, 82 p.

Curtet A., 1959, "Les dolmens des Deux-Sèvres, remarques sur leur distribution géographique ", Bulletin de la Société préhistorique française, 56, 3, p. 236-241.

Descola P., 2005, Par-delà nature et culture, Paris, Gallimard, coll. "Bibliothèque des Sciences Humaines ", 623 p.

Fairén Jiménez S., Cruz Berrocal M., López-Romero González de la Aleja E. et Walid Sbeinati S., 2006, "Las vías pecuarias como elementos arqueológicos ", dans Grau Mira I. (dir.), La aplicación de los SIG en la arqueología del paisaje, p. 55-68.

Gaudin L., 2004, Transformations spatio-temporelles de la végétation du nord-ouest de la France depuis la fin de la dernière glaciation. Reconstitution paléo-paysagères, Rennes, université de Rennes 1, 763 p.

Gaudin L., Onnée Y. et Paris F., 2000, « Préhistoire du pays de La Bouëxière (35). Mégalithes connus et inconnus au bois de la Mancellière ", Les Dossiers du Centre Régional d'Archéologie d'Alet, 28, p. 91-106.

Germond G., 2001, Les Deux-Sèvres préhistoriques, La Crèche, Geste éditions, $316 \mathrm{p}$.

Ghesquière E. et Guyodo J.-N., 2008, " Les industries lithiques taillées des $\mathrm{IV}^{\mathrm{e}}$ et $\mathrm{III}^{\mathrm{e}}$ millénaires av. J.-C. dans le quart nord-ouest de la France ", dans Dias-Meirinho M.-H., Léa V., Gernigon K., Fouéré P., Briois F. et BAILly M. (dir.), Les industries lithiques taillées des IVe et III millénaires en Europe occidentale : colloque international (Toulouse, 7-9 avril 2005), Oxford, John and Erica Hedges Ltd. (British Archaeological Reports, International Series), p. 113-133.

Giot P.-R., 1960, «Informations archéologiques. Circonscription de Rennes ", Gallia Préhistoire, 3, 1, p. 157-169.

Giot P.-R., 1965, "Informations archéologiques. Circonscription de Rennes », Gallia Préhistoire, 8, 1, p. 33-50.

Giot P.-R., 1958, «Informations archéologiques. Circonscription de Rennes ", Gallia Préhistoire, 1, 1, p. 122-133.

Gouletquer P.-L., 1979, Préhistoire du Futur, Bretagnes, Morlaix, $111 \mathrm{p}$.

Grancière A. de la et Harmois A.-L., 1916, "Inventaire des Haches-Marteaux et des Haches doubles ou Casse têtes en Pierre polie, trouvées en Bretagne-Armorique. (Suite) ", Bulletin de la Société préhistorique française, 13, 5, p. 280-288. 
Guilaine J., Besse M., Lemercier O., Salanova L., Strahm C. et Vander Linden M., 2004, « Avant-propos : les Campaniformes aujourd'hui », Bulletin de la Société préhistorique française, 101, 2, p. 197-200.

Hamon A.-L., 2010, Vitré, Ille-et-Vilaine, Boulevard de Laval. La ferme laténienne de la Grande Haie, Rennes, SRA Bretagne/Inrap, $243 \mathrm{p}$.

HÉnAfF X., 2002, Les habitats néolithiques en Bretagne, Rennes, Institut culturel de Bretagne et Association des Travaux du Laboratoire d'Anthropologie, 95 p.

Hinguant S., Cassen S., Audren C., Lannuzel G. et MarCHAND G., 1998, "L'habitat Villeneuve-Saint-Germain du Haut-Mée (Saint-Étienne-en-Coglès, Ille-et-Vilaine) ", Bulletin de la Société préhistorique française, 95, 1, p. 41-76.

Ihuel E., 2012, « La circulation des lames dans le Massif armoricain au Néolithique », dans Marchand G. et Querré G. (dir.), Roches et sociétés en préhistoire entre massifs cristallins et bassins sédimentaires, Rennes, Presses universitaires de Rennes, coll. «Archéologie \& Culture », p. 325-340.

Joussaume R. et Laporte L., 2006, « Monuments funéraires néolithiques dans l'ouest de la France ", dans Joussaume R., Laporte L. et Scarre C. (dir.), Origine et développement $d u$ mégalithisme de l'Ouest de l'Europe, Niort, Conseil général des Deux-Sèvres, p. 319-343.

Juhel L., 2010, Louvigné-de-Bais, Torcé, Étrelles, Argentré-duPlessis, Ille-et-Vilaine, LGV-Secteur 3. Occupations du Néolithique à l'Époque moderne sur le tracé de la LGV Rennes-Le Mans, Rennes, SRA Bretagne/Inrap, 233 p.

Kerdivel G., 2019a, Haches polies à Juvigné, Groupe de Recherches archéologiques de la Mayenne (GRAM), document en ligne : [http://gram.over-blog.com/2019/04/ haches-polies-a-juvigne.html].

Kerdivel G., 2019b, Les haches d'un mystérieux don Chassin conservées au musée d'Ernée, Groupe de Recherches archéologiques de la Mayenne (GRAM), document en ligne : [http:// gram.over-blog.com/2019/06/les-haches-d-un-mysterieuxdon-chassin-conservees-au-musee-d-ernee.html].

Kerdivel G., 2019c, Une hache-marteau à Cuillé, Groupe de Recherches archéologiques de la Mayenne (GRAM), document en ligne : [http://gram.over-blog.com/2019/04/unehache-marteau-a-cuille.html].

Kerdivel G., 2012, Occupation de l'espace et gestion des ressources à l'interface massifs anciens/bassins secondaires et tertiaires. L'exemple du Massif armoricain et de ses marges au Néolithique, Oxford, Archaeopress (British Archaeological Reports, International Series, 2383), 362 p.

Kerdivel G., 2014, "Quelques remarques d'archéologie spatiale concernant la distribution géographique des enceintes néolithiques du Centre-Ouest de la France et des marges du Massif armoricain ", dans Joussaume R., Large J.-M., Corson S., Le Meur N. et Tortuyaux J.-P. (dir.), Enceintes néolithiques de l'Ouest de la France de la Seine à la Gironde,
Chauvigny, Association des Publications Chauvinoises, "Mémoire ", XLVIII, p. 273-290.

Kerdivel G., Bénéteaud L., Bézos A., Bodinier B. et Jude F., 2017, "Produire des lames de hache en dolérite au Néolithique en Mayenne. La carrière-atelier de Beulin à Saint-Germain-le-Guillaume ", Bulletin de la Société préhistorique française, 114, 4, p. 659-690.

Kerdivel G. et Hamon G., 2010, «Un site du Néolithique moyen, du Néolithique final et de l'âge du Fer à la Burette à Banville (Calvados) ", dans Billard C. et Legris M. (dir.), Premiers Néolithiques de l'Ouest de la France. Cultures, réseaux, échanges des premiers Néolithiques à leur expansion, Rennes, Presses universitaires de Rennes, coll. « Archéologie \& Culture ", p. 211-235.

Laporte L., Daire M.-Y., Kerdivel G. et López Romero GonzÁlez de la Aleja E., 2015, «Back and forward: Neolithic Standing Stones and Iron Age Stelae in French Brittany ", dans Díaz-Guardamino M., García Sanjuan L. et Wheatley D. (dir.), The Lives of Prehistoric Monuments in Iron Age, Roman and Medieval Europe, Oxford, Oxford University Press, p. 141-161.

Laporte L., Guyodo J.-N., Bizien-Jaglin C., Bernard V., Bertin F., Blanchet S., Dietsch-Sellami M.-F., Guitton V., Hamon A.-L., Hamon G., Lemouland Q., LucQuin A., Noslier A. et Quesnel L., 2007, « Nouvelles découvertes en milieu humide autour de l'habitat ceinturé du Néolithique moyen à Lillemer (Ille-et-Vilaine, France) ", dans Besse M. (dir.), Sociétés néolithiques. Des faits archéologiques aux fonctionnements socio-économiques, Lausanne, coll. "Cahiers d'Archéologie Romande », p. 341-349.

Le Boulanger F., 2012, Torcé (Ille-et-Vilaine), La Petite Gatellerie. Indices d'occupation du Néolithique et du haut Moyen Âge, Rennes, SRA Bretagne/Inrap, 56 p.

Le Boulanger F., 2002, Visseiche "La Grande Censerie " (35359) (Ille-et-Vilaine), Rennes, SRA Bretagne/Inrap, 25 p.

Le Cloirec G., 2005, Bais (Ille-et-Vilaine). Carrière des Vallons, Rennes, SRA Bretagne/Inrap, $29 \mathrm{p}$.

Le Gall J., 2015, Ille-et-Vilaine, Ossé, la Claraiserie (tracé de la $L G V$ "Rennes-Le Mans", secteur 2). Une exploitation agricole de la fin du second âge du Fer et du début de l'Antiquité (II s. av. n. è.-ler s. d. n. è.), Rennes, SRA Bretagne/Inrap, $268 \mathrm{p}$.

Le Roux C.-T., 1998, "La Roche-aux-Fées en Essé ", Bulletin et mémoires de la Société archéologique du département d'Illeet-Vilaine, 101, p. 37-47.

Le Roux C.-T., 1999, L'outillage de pierre polie en métadolérite du type A. Les ateliers de Plussulien (Côtes-d'Armor) : production et diffusion au Néolithique dans la France de l'Ouest et au-delà, Rennes, Association des Travaux du Laboratoire d'Anthropologie, coll. "Travaux du Laboratoire ", 43, 244 p.

Leroux G., 2003, Bruz (35), Cicé, Rennes, SRA Bretagne/ Inrap, $42 \mathrm{p}$. 
Leroux G., 2015, Piré-sur-Seiche, Ille-et-Vilaine, ZAC de Bellevue, secteur est. Un itinéraire routier de l'âge du Bronze au Moyen Âge, Rennes, SRA Bretagne/Inrap, 152 p.

Leroux G., 1997, Prospection-inventaire dans le bassin oriental de la Vilaine. Archéologie aérienne dans les arrondissements de Fougères, Redon, Rennes, Vitré (Ille-et-Vilaine), Rennes, SRA Bretagne/Inrap, $246 \mathrm{p}$.

Leroux G., 1999, Prospection-inventaire dans le bassin oriental de la Vilaine. Archéologie aérienne dans les arrondissements de Fougères, Redon, Rennes, Vitré (Ille-et-Vilaine), Rennes, SRA Bretagne/Inrap, $236 \mathrm{p}$.

Letterlé F., 1986, "Le monument mégalithique des Erves à Sainte-Suzanne (Mayenne) et ses implications chronologiques ", dans Aubin G. (dir.), Actes du $10^{\circ}$ colloque interrégional sur le Néolithique (Caen, 30 septembre-2 octobre 1983), Rennes, Association pour la Diffusion des Recherches archéologiques dans l'Ouest de la France, supplément, p. 149-164.

L'Helgouac'H J., 1965, Les sépultures mégalithiques en Armorique, Rennes, université de Rennes, 330 p.

Marcigny C. et Ghesquière E. (dir.), 2003, Lîle Tatihou (Manche) à l'âge du Bronze. Habitats et occupation du sol, Paris, Éditions de la Maison des sciences de l'homme, Document d'archéologie française, 96, $192 \mathrm{p}$.

Marsille L., 1911, "Note archéologique. I Le polissoir de Sarzeau ", Bulletin de la Société polymathique du Morbihan, 55, p. 194-199.

Meuret J.-C., 1986, Peuplement à l'est de l'Ille-et-Vilaine entre Vilaine et Semnon. Préhistoire et protohistoire, Mémoire de maîtrise, Rennes, université de Haute-Bretagne, 185 p.

Meuret J.-C., 1993, Peuplement, pouvoir et paysage sur la marche Anjou-Bretagne (des origines aux Moyen Âge), supplément à La Mayenne : Archéologie, Histoire, 4, Laval, Société d'Archéologie et d'Histoire de la Mayenne, 656 p.

Nicolas E., Marchand G., Hénaff X., Juhel L., Pailler Y., Darboux J.-R. et Errera M., 2013, « Le Néolithique ancien à l'ouest de la Bretagne : nouvelles découvertes à Pen Hoat Salaün (Pleuven, Finistère) ", L'Anthropologie, 117, 2, p. 195-237.

O'Kelly M. J., 2001, Early Ireland. An introduction to Irish Prehistory, Cambridge, Cambridge University Press, 375 p.

Onnée Y., Ory R. et Gaudin L., 2000, «Prospections lithiques sur la commune de La Bouëxière (35) ", Les Dossiers du Centre Régional d'Archéologie d'Alet, 28, p. 107-116.

Pailler Y., 2009, " Neolithic Fibrolite Working in the West of France ", dans O'Connor B., Cooney G. et Chapman J. (dir.), Materialitas: Working Stone, Carving Identity, Oxbow Books, "Prehistoric Society Research Papers ", 3, p. 113126.

Pailler Y., Marchand G., Blanchet S., Guyodo J.-N. et Hamon G., 2008, " Le Villeneuve-Saint-Germain dans la péninsule armoricaine : les débuts d'un enquête ", dans
Burnez-Lanotte L., Illet M. et Allard P. (dir.), Fin des traditions danubiennes dans le Néolithique du Bassin parisien et de la Belgique (5100-4700 av. J.-C.). Autour des recherches de C. Constantin, Paris, Société préhistorique française, "Mémoires", 44, p. 91-111.

Pelegrin J., 2012, "Observations sur la taille et le polissage de haches en silex ", dans De Labriffe P.-A. et Thirault E. (dir.), Produire des haches au Néolithique : de la matière première à l'abandon, Paris, Société préhistorique française, p. 87-106.

Poullle D., 2011, Bais (Ille-et-Vilaine) - Bourg Saint-Pair. Un domaine rural de la campagne des Riedones, Rennes, SRA Bretagne/Inrap, $524 \mathrm{p}$.

Pouille D., 2004, Corps-Nuds. ZAC des Grands Sillons $I^{\text {re }}$ tranche (Ille-et-Vilaine), SRA Bretagne/Inrap, 19 p.

Pouille D. et Le Cloirec G., 1992, Rennes-Metro VAL. Sondages archéologiques, Rennes, SRA Bretagne/Afan, 70 p.

Priol A., 1997, Rapport de prospection-inventaire 1997, Bassin de Rennes, $17 \mathrm{p}$.

Provost A., 1989, La prospection-inventaire du bassin de Rennes 1988-1989, Rennes, SRA Bretagne/CERAPAR, 732 p.

Provost A., Champagne F. et Le Bouteiller P., 1985, La villa gallo-romaine de la Guyaumerais à Châtillon-sur-Seiche, Rennes, SRA Bretagne, 210 p.

Scarre C., 2001, "Modeling Prehistoric Populations: The Case of Neolithic Brittany ", Journal of Anthropological Archaeology, 20, 3, p. 285-313.

Scotт J.-C., 2019, "Homo Domesticus». Une histoire profonde des premiers États, Paris, La Découverte, 301 p.

Sellier D., 2017, "Le dolmen de la Roche-aux-Fées à Essé (Ille-et-Vilaine) et son environnement géomorphologique : approche géoarchéologique ", Revue archéologique de l'Ouest, 34, p. 23-47.

SiCARD S., 2016, Bretagne, Ille-et-Vilaine, Rannée. Les Rimbaudières. Les occupations protohistoriques des Rimbaudières, Rennes, Inrap, 126 p.

Simier B., 2016, Noyal-Châtillon-sur-Seiche (Ille-et-Vilaine), Illot Floratrait. Pars rustica de la villa de la Guyomerais et voie secondaire antique, Rennes, SRA Bretagne/Inrap, $392 \mathrm{p}$.

Tinévez J.-Y. (dir.), 2004, Le site de la Hersonnais à Pléchâtel (Ille-et-Vilaine) : un ensemble de bâtiments collectifs du Néolithique final, Paris, Société préhistorique française, "Travaux ", 5, $172 \mathrm{p}$.

Toron S., 2016, La Guerche-de-Bretagne (35), La Pinelière. Site $4-R D$ 178. Déviation La Guerche-de-Bretagne et Rannée, SRA Bretagne/Éveha, 114 p.

Trautmann I., Becq-Giraudon J.-F. et Carn A., 1994, Carte et notice explicative, Carte géol. France (1/50000), feuille Janzé (353), Orléans, BRGM, 79 p.

Trautmann I. et Carn A., 1997, Carte et notice explicative, Carte géol. France (1/50 000), feuille La Guerche-de-Bretagne (353), Orléans, BRGM, 67 p. 
Trautmann I., Lacquement F., Vernhet Y. et Pivette B., 2011, Carte et notice explicative, Carte géol. France (1/50000), feuille Vitré (318), Orléans, BRGM, 133 p.

Valais A., Meuret J.-C., Nauleau J.-F., Edin F., Hamon G. et Muttarelli E., 2008, "Le Clos-Henry : une ferme de La Tène finale à Château-Gontier (Mayenne) ", Revue archéologique de l'Ouest, 25, p. 139-161.
Whittle A., 2001, "From mobility to sedentism: change by degrees ", dans Kertész R. et Makkay J. (dir.), From the Mesolithic to the Neolithic, Proceedings of the International Archaeological Conference held in the Damjanich Museum of Szolnok (September 22-27, 1996), Budapest, Archaeolingua Alapitvany, p. 47-461.

\section{Das Tal der Seiche und das obere Tal der Vilaine vom Beginn des Neolithikums bis zum Chalkolithikum: Bilanz und Forschungsperspektiven}

Dieser Beitrag stellt den aktuellen Forschungsstand zum Neolithikum im oberen Tal der Vilaine und im Tal der Seiche dar. Dabei wird auch das Tal der Blosne miteinbezogen. Alle drei Flusstäler befinden sich im Osten der Bretagne, im Herzen des amorikanischen Massivs. Bei dieser Gelegenheit sind mehrere Neuentdeckungen mitzuteilen: einige Hinweise auf eine Besiedlung der Villeneuve-Saint-Germain-Kultur (VSG), eine mögliche Grabanlage vom Typ Passy, eine Fundstelle, an der Mikrogranit gewonnen wurde und die Herstellung von Beilen aus diesem Material, die ins Spätneolithikum datiert werden, sowie zahlreichen Beilklingen, die bisher weitgehend unbekannt waren, etc.

Die Zuordnung zu einem Umweltkontext und die entsprechende chronologische Einordnung konnten zeigen, dass die verfügbaren archäologischen Daten weitgehend dem entsprechen, was man bisher aus den Randgebieten des amorikanischen Massivs kennt, insbesondere eine Zunahme der Siedlungsfundstellen in der letzten Phase des Neolithikums sowie einen zunehmenden Druck auf die natürlichen Umweltverhältnisse. Alles zusammengenommen erlauben diese neuen Erkenntnisse, deutlicher wahrzunehmen, was wir derzeit alles noch nicht wissen und daraus künftige Forschungsprojekte zu entwickeln.

Schlagwortindex: Bretagne, Neolithikum, polierte Beile, Siedlung, Menhir, Prospektion, räumliche Archäologie, Besiedlungsmodell, Siedlungsraum.

\section{El valle del Seiche y la cuenca alta del Vilaine desde los inicios del Neolítico al Calcolítico: balance y perspectivas}

Este articulo hace un balance sobre el Neolítico en la cuenca alta del rio Vilaine y del Seiche incluyendo la del Blosne, todas situadas en el este de Bretaña, en el corazón del macizo Armoricano. En este contexto, se señalan varios hallazgos: algunos indicios del periodo Villeneuve-Saint-Germain, una posible tumba de tipo Passy, una cantera de microgranito y un taller de fabricación de hachas del mismo material, atribuidos al Neolitico final, numerosas hachas inéditas, etc. El estudio del contexto medioambiental desde una perspectiva cronológica permite demostrar que los datos disponibles se insertan en el conocimiento actual de los márgenes del macizo Armoricano, con un aumento del número de yacimientos en la última fase del Neolitico y una presión creciente sobre el medioambiente. Este balance permite sobre todo apreciar las lagunas y proponer proyectos para el futuro.

Palabras clave: Bretaña, Neolitico, hacha pulimentada, poblado, menhir, prospección, arqueología espacial, modelo de poblamiento, ocupación del territorio. 
